\title{
Hilbert Space Theory for Reflectionless Relativistic Potentials
}

\author{
By
}

Simon N. M. RuiJSEnAARS*

\begin{abstract}
We study Hilbert space aspects of explicit eigenfunctions for analytic difference operators that arise in the context of relativistic two-particle Calogero-Moser systems. We restrict attention to integer coupling constants $g / \hbar$, for which no reflection occurs. It is proved that the eigenfunction transforms are isometric, provided a certain dimensionless parameter $a$ varies over a bounded interval $\left(0, a_{\max }\right)$, whereas isometry is shown to be violated for generic $a$ larger than $a_{\max }$. The anomaly is encoded in an explicit finite-rank operator, whose rank increases to $\infty$ as $a$ goes to $\infty$.
\end{abstract}

\section{Contents}

$\S 1 . \quad$ Introduction

$\S 2 . \quad$ The General Framework

$\S 3 . \quad$ The Repulsive Regime

$\S 4$. The Attractive Regime

$\S 5$. The Extra Regime

Appendix A. Proofs of Theorems 2.1-2.3

References

Communicated by T. Kawai, April 5, 2000

2000 Mathematics Subject Classification(s): 81Q10, 47B39, 33E30

*Work partly supported by the Netherlands Organisation for the Advancement of Research(NWO).

${ }^{*}$ Centre for Mathematics and Computer Science, P.O.Box 94079, 1090 GB Amsterdam, The Netherlands. 


\section{§1. Introduction}

The $M$-particle relativistic Calogero-Moser system involves the commuting analytic difference operators

$$
\begin{aligned}
S_{ \pm k} \equiv \sum_{\substack{I \subset\{1, \ldots, M\} \\
|I|=k}} \prod_{\substack{c \in I \\
j \notin I}} f_{\mp}\left(x_{\imath}-x_{j}\right) \cdot \exp \left(\mp i \frac{\hbar}{m c} \sum_{l \in I} \partial_{l}\right) \cdot \\
\prod_{\substack{\imath \in I \\
j \notin I}} f_{ \pm}\left(x_{i}-x_{j}\right), \quad k=1, \ldots, M,
\end{aligned}
$$

where the 'potential functions' $f_{ \pm}(x)$ are given by

$$
f_{ \pm}(x) \equiv(\sigma(x \pm i g / m c) / \sigma(x))^{1 / 2},
$$

with $\sigma(x)$ the Weierstrass $\sigma$-function. Specifically, the time and space translation generators are the Hamiltonian and total momentum operator

$$
\begin{aligned}
H_{\text {rel }} & \equiv m c^{2}\left(S_{1}+S_{-1}\right), \\
P_{\text {rel }} & \equiv m c\left(S_{1}-S_{-1}\right),
\end{aligned}
$$

which together with the boost generator

$$
B \equiv-m \sum_{j=1}^{M} x_{j}
$$

give rise to a representation of the Lie algebra of the Poincaré group,

$$
\left[H_{\mathrm{rel}}, P_{\mathrm{rel}}\right]=0, \quad\left[H_{\mathrm{rel}}, B\right]=i \hbar P_{\mathrm{rel}}, \quad\left[P_{\mathrm{rel}}, B\right]=i \hbar H_{\mathrm{rel}} / c^{2} .
$$

The nonrelativistic limit $c \rightarrow \infty$ yields

$$
H_{\mathrm{rel}}=M m c^{2}+H_{\mathrm{nr}}+O\left(c^{-2}\right),
$$

where $H_{\mathrm{nr}}$ is the nonrelativistic Calogero-Moser Hamiltonian,

$$
H_{\mathrm{nr}} \equiv-\frac{\hbar^{2}}{2 m} \sum_{j=1}^{M} \partial_{\jmath}^{2}+\frac{g(g-\hbar)}{m} \sum_{1 \leq j<k \leq M} \wp\left(x_{j}-x_{k}\right),
$$

with $\wp(x)$ the Weierstrass $\wp$-function. Moreover, one readily verifies

$$
P_{\mathrm{rel}}=P_{\mathrm{nr}}+O\left(c^{-2}\right), \quad P_{\mathrm{nr}} \equiv \frac{\hbar}{i} \sum_{j=1}^{M} \partial_{j}
$$


Obviously, the resulting Galilei group Lie algebra representation

$$
\left[H_{\mathrm{nr}}, P_{\mathrm{nr}}\right]=0, \quad\left[H_{\mathrm{nr}}, B\right]=i \hbar P_{\mathrm{nr}}, \quad\left[P_{\mathrm{nr}}, B\right]=i \hbar M m
$$

is satisfied when $\wp(x)$ is replaced by an arbitrary potential $V(x)$. By contrast, the replacement of the $\sigma$-function in (1.2) by other functions would yield a non-zero commutator for $H_{\text {rel }}$ and $P_{\text {rel }}$ when $M>2$, precluding a relativistic interpretation.

The integrable one-dimensional $M$-particle systems just described were introduced at the classical level in a joint paper with H. Schneider [1] and at the quantum level in our paper Ref. [2]. The main inspiration for arriving at these systems came from the question whether a relativistic point particle dynamics describing the solitons/antisolitons/breathers in the relativistic sine-Gordon field theory exists. There is meanwhile considerable evidence that this problem can be solved via the above hyperbolic systems (obtained by specializing $\sigma$ to sinh), and the present paper yields in particular a further confirmation of this scenario.

Both the classical and the quantum relativistic systems have been encountered in various other contexts and have been studied from a great many viewpoints. We refer to our lecture notes Ref. [3] for a detailed survey and bibliographical information until 1995. More recent work includes for example Refs. [4]-[44], from which further pertinent articles can be traced.

This paper may be viewed as a sequel to our recent paper [45], where we studied eigenfunctions of the above (reduced) two-particle Hamiltonian with hyperbolic interactions. In the latter paper we focused on properties of an algebraic character. Here, we consider Hilbert space properties of the pertinent eigenfunctions and operators for a subset of the parameter space allowed in Ref. [45]. Apart from the repulsive parameter regime, we study a closely related attractive regime, and an extra (Dirac type) regime that has no analog in the nonrelativistic setting.

To begin with the latter setting, the repulsive and attractive Hamiltonians can be taken to be

$$
\begin{aligned}
& H_{r}^{(\mathrm{nr})} \equiv \frac{1}{2 m}\left(-\hbar^{2} \frac{d^{2}}{d x^{2}}+\frac{g(g-\hbar) \nu^{2}}{\operatorname{sh}^{2}(\nu x)}\right), \\
& H_{a}^{(\mathrm{nr})} \equiv \frac{1}{2 m}\left(-\hbar^{2} \frac{d^{2}}{d x^{2}}-\frac{g(g-\hbar) \nu^{2}}{\operatorname{ch}^{2}(\nu x)}\right) .
\end{aligned}
$$

Thus they are related by the crossing substitution $x \rightarrow x+i \pi / 2 \nu$. Choosing $g \in \hbar \mathbb{N}$, one winds up with reflectionless eigenfunctions, and in this paper 
we restrict ourselves to the corresponding choice of coupling constants in the relativistic framework.

Specifically, the three Hamiltonians are the analytic difference operators (from now on abbreviated as $\mathrm{A} \Delta \mathrm{Os}$ )

$$
\begin{aligned}
& H_{r} \equiv\left(\frac{\operatorname{sh} \nu(x-i g \beta)}{\operatorname{sh} \nu x}\right)^{1 / 2} T_{i \hbar \beta}^{x}\left(\frac{\operatorname{sh} \nu(x+i g \beta)}{\operatorname{sh} \nu x}\right)^{1 / 2}+(i \rightarrow-i), \\
& H_{a} \equiv\left(\frac{\operatorname{ch} \nu(x-i g \beta)}{\operatorname{ch} \nu x}\right)^{1 / 2} T_{\imath \hbar \beta}^{x}\left(\frac{\operatorname{ch} \nu(x+i g \beta)}{\operatorname{ch} \nu x}\right)^{1 / 2}+(i \rightarrow-i), \\
& H_{e} \equiv\left(\frac{\operatorname{ch} \nu(x-i g \beta)}{\operatorname{ch} \nu x}\right)^{1 / 2} T_{\imath \hbar \beta}^{x}\left(\frac{\operatorname{ch} \nu(x+i g \beta)}{\operatorname{ch} \nu x}\right)^{1 / 2}-(i \rightarrow-i) .
\end{aligned}
$$

(Here and below, a formula of the form $F(i) \pm(i \rightarrow-i)$ stands for $F(i) \pm F(-i)$.) The parameters are restricted by

$$
\hbar, \beta \equiv 1 / m c, \nu \in(0, \infty), \quad g / \hbar=N+1 \in \mathbb{N}^{*},
$$

and $T_{ \pm \imath \hbar \beta}^{x}$ are the translation operators defined by

$$
\left(T_{\alpha}^{z} f\right)(z) \equiv f(z-\alpha), \quad \alpha \in \mathbb{C}
$$

Therefore, $H_{a}$ is again related to $H_{r}$ by taking $x \rightarrow x+i \pi / 2 \nu$, whereas the relation of $H_{e}$ to $H_{r}$ will be clarified later on.

The operators $H_{r}, H_{a}$ and $H_{e}$ are formally self-adjoint on the Hilbert space $L^{2}(\mathbb{R}, d x)$. As will transpire below, this formal property is a poor guide. Indeed, an important aspect of this paper is that it makes clear (by explicit examples) that a general eigenfunction expansion theory for analytic difference operators must cope with new phenomena not present for discrete difference and differential operators. To date, no such theory exists, in contrast to the Weyl-KodairaTitchmarsh theory for the latter operator classes. (See, e.g., Refs. [46]-[51] for accounts of WKT theory from various complementary viewpoints.) The special cases studied here and in our related papers Refs. [52, 53] suggest that one should first of all try and isolate some general criteria guaranteeing that a well-behaved eigenfunction transform exists.

The key problem with eigenfunctions of $\mathrm{A} \Delta \mathrm{O}$ s such as (1.13)-(1.15) is that they are highly non-unique. Indeed, they can be multiplied by arbitrary functions with period $i \hbar \beta$. This problem can be ignored for A $\Delta \mathrm{O}$ s that can be defined as self-adjoint Hilbert space operators by restricting attention to eigenfunctions that are (in essence) polynomials - a property destroyed upon 
multiplication by a non-constant $i \hbar \beta$-periodic function. But for the above hyperbolic $\mathrm{A} \Delta \mathrm{Os}$ this avenue is closed. (Their trigonometric versions, however, can be handled in terms of Askey-Wilson polynomials.)

As it turns out, the infinite-dimensional eigenfunction space of the $\mathrm{A} \Delta \mathrm{Os}$ $H_{r}, H_{a}$ and $H_{e}$ can be reduced to a two-dimensional one by insisting on an additional eigenfunction property for an $\mathrm{A} \Delta \mathrm{O}$ in their (formal) commutant. For the $g=(N+1) \hbar$ case considered in this paper this operator may be taken to be the 'free' $\mathrm{A} \Delta \mathrm{O}$

$$
A \equiv T_{i \pi / \nu}^{x}+T_{-i \pi / \nu}^{x} .
$$

It is these joint eigenfunctions that can be used to associate to $H_{r}, H_{a}$ and $H_{e}$, as well as to $A$, bona fide self-adjoint Hilbert space operators. The latter are denoted by the same symbols, but it should be stressed that the Hilbert space operator $A$ depends not only on the case at hand, but also on $\beta$ and $N$. This dependence shows up in the associated $S$-operator; the crux is that the definition domain of $A$ varies.

To describe these Hilbert space results in more detail, let us denote from now on the even and odd subspaces of $L^{2}(\mathbb{R})$ by $L_{+}^{2}(\mathbb{R})$ and $L_{-}^{2}(\mathbb{R})$, resp. Then we obtain self-adjoint operators on the following Hilbert spaces, provided the dimensionless product parameter

$$
a \equiv \hbar \beta \nu \in(0, \infty)
$$

is restricted as indicated:

$$
\begin{gathered}
L_{-}^{2}(\mathbb{R}, d x), \quad a \in(0, \pi / N), \quad\left(H_{r}\right), \\
L^{2}(\mathbb{R}, d x), \quad a \in(0, \pi / 2 N), \quad\left(H_{a}, H_{e}\right) .
\end{gathered}
$$

To be specific, we obtain an isometric eigenfunction transform

$$
\mathcal{F}_{r,-}: L_{-}^{2}(\mathbb{R}, d p) \rightarrow L_{-}^{2}(\mathbb{R}, d x)
$$

onto $L_{-}^{2}(\mathbb{R}, d x)$, conjugating $H_{r}$ and $A$ to multiplication by $2 \operatorname{ch} \beta p$ and $2 \operatorname{ch} \pi p / \hbar \nu$, resp. Similarly, we obtain isometric eigenfunction transforms

$$
\mathcal{F}_{s}: L^{2}(\mathbb{R}, d p) \rightarrow L^{2}(\mathbb{R} . d x), \quad s=a, e,
$$

conjugating $A$ to multiplication by $2 \operatorname{ch} \pi p / \hbar \nu$, and $H_{a}$ and $H_{e}$ to multiplication by $2 \operatorname{ch} \beta p$ and $2 \operatorname{sh} \beta p$, resp. (Note $H_{r}$ and $H_{a}$ formally commute with parity, whereas $H_{e}$ anticommutes.) The operator $\mathcal{F}_{e}$ maps onto $L^{2}(\mathbb{R}, d x)$, while the 
range of $\mathcal{F}_{a}$ equals the orthocomplement of $N$ pairwise orthogonal bound states with eigenvalues

$$
2 \cos (k a), \quad k=1, \ldots, N, \quad\left(H_{a} \text { - eigenvalues }\right)
$$

and corresponding parity $(-)^{N-k}$.

At this point it should be emphasized once again that the domains and actions of the self-adjoint Hilbert space operators we associate to the A $\Delta \mathrm{Os}$ $H_{r}, H_{a}, H_{e}$ and $A$ are defined indirectly, via the isometries $\mathcal{F}_{r,-}, \mathcal{F}_{a}$ and $\mathcal{F}_{e}$. This is in sharp contrast to the situation for ordinary differential and discrete difference operators, where one typically defines the operator at first as a symmetric operator on a dense subspace, and then studies eventual self-adjoint extensions. The examples studied here and in our previous paper [52] strongly suggest that the latter approach is not as fruitful and revealing in the $\mathrm{A} \Delta \mathrm{O}$ setting. In particular, our results illustrate in a quite concrete way that the 'free' $\mathrm{A} \Delta \mathrm{O} A(1.18)$ can be defined as an essentially self-adjoint operator with the natural $(\mathrm{A} \Delta \mathrm{O})$ action on an infinite-dimensional family of dense subspaces, whose pairwise intersection is the zero vector. (Cf. especially the paragraph above Theorem 2.2.)

The above 'constructive' results are supplemented by a number of 'destructive' ones. In particular, we prove that in the repulsive case isometry and self-adjointness break down on $L_{+}^{2}(\mathbb{R}, d x)$ for generic $a \in(0, \infty)$ and on $L_{-}^{2}(\mathbb{R}, d x)$ for generic $a$ outside $(0, \pi / N)$. Similarly, these anomalies are shown to arise in the attractive and extra cases for generic $a$ in $[\pi / 2 N, \infty)$. The isometry obstructions are encoded in finite-dimensional subspaces whose dimension (generically) increases as $a$ increases.

We proceed by detailing the pertinent eigenfunctions and some of their features. As a preliminary, we introduce weight functions

$$
\begin{aligned}
& w_{s}(y) \equiv\left(\prod_{j=1}^{N} 4 \operatorname{sh}(y+i j a) \operatorname{sh}(y-i j a)\right)^{-1}, \\
& w_{c}(y) \equiv\left(\prod_{\jmath=1}^{N} 4 \operatorname{ch}(y+i j a) \operatorname{ch}(y-i j a)\right)^{-1} .
\end{aligned}
$$

These functions can be used to conjugate the $\mathrm{A} \Delta \mathrm{Os}$ (1.13)-(1.15) to $\mathrm{A} \Delta \mathrm{Os}$ with meromorphic coefficients. Specifically, one has

$$
B_{r} \equiv w_{s}(\nu x)^{-1 / 2} H_{r} w_{s}(\nu x)^{1 / 2}=\frac{\operatorname{sh}(\nu x+i N a)}{\operatorname{sh} \nu x} T_{i \hbar \beta}^{x}+(i \rightarrow-i),
$$




$$
\begin{aligned}
& B_{a} \equiv w_{c}(\nu x)^{-1 / 2} H_{a} w_{c}(\nu x)^{1 / 2}=\frac{\operatorname{ch}(\nu x+i N a)}{\operatorname{ch} \nu x} T_{\imath \hbar \beta}^{x}+(i \rightarrow-i), \\
& B_{e} \equiv w_{c}(\nu x)^{-1 / 2} H_{e} w_{c}(\nu x)^{1 / 2}=\frac{\operatorname{ch}(\nu x+i N a)}{\operatorname{ch} \nu x} T_{\imath \hbar \beta}^{x}-(i \rightarrow-i),
\end{aligned}
$$

as is readily checked. The same similarity transformations on $A$ (1.18) yield

$$
B=(-)^{N}\left(T_{\imath \pi / \nu}^{x}+T_{-\imath \pi / \nu}^{x}\right)
$$

(To appreciate how the factor $(-)^{N}$ arises, take $x \rightarrow \pm \infty$.)

The $H_{s}$-eigenfunctions, $s=r, a, e$, are now given by

$$
\begin{aligned}
& F_{r}(\nu, \beta ; x, p) \equiv w_{s}(\nu x)^{1 / 2} E_{r}(\nu, \beta ; x, p) w_{s}(\beta p)^{1 / 2}, \\
& F_{a}(\nu, \beta ; x, p) \equiv w_{c}(\nu x)^{1 / 2} E_{a}(\nu, \beta ; x, p) w_{s}(\beta p)^{1 / 2}, \\
& F_{e}(\nu, \beta ; x, p) \equiv w_{c}(\nu x)^{1 / 2} E_{c}(\nu, \beta ; x, p) w_{c}(\beta p)^{1 / 2},
\end{aligned}
$$

where the entire $B_{s}$-eigenfunctions $E_{s}, s=r, a, e$, are of the form

$$
\begin{gathered}
E_{s}(\nu, \beta: x, p) \equiv \exp (i x p / \hbar) P^{(s)}\left(e^{\nu x}, e^{\beta p}\right), \\
P^{(s)}(y, z) \equiv \sum_{m \cdot n=0}^{N} c_{m n}^{(s)} y^{N-2 m} z^{N-2 n}
\end{gathered}
$$

The numbers $c_{m n}^{(r)}$ are Laurent polynomials in the phase

$$
q \equiv \exp (i a), \quad a=\hbar \beta \nu
$$

with coefficients in $\mathbb{N}$ after multiplication by $(-)^{m+n} i^{N}$ :

$$
\begin{gathered}
c_{m n}^{(r)} \equiv(-)^{m+n}(-i)^{N} q^{N(N+1) / 2} c_{m} \sum_{\substack{-N \leq l_{1}<\cdots<l_{n} \leq N \\
l_{J} \notin\{-N+m \cdots \cdots m\}}} q^{-2\left(l_{1}+\cdots+l_{n}\right)}, \\
c_{m} \equiv \sum_{1 \leq k_{1}<\cdots<k_{m} \leq N} q^{-2\left(k_{1}+\cdots+k_{m}\right)} .
\end{gathered}
$$

(Here, empty sums are defined to be 1 ; note $c_{m}$ is in essence a $q$-binomial coefficient, cf. Ref. [54].) Moreover,

$$
\begin{aligned}
& c_{m n}^{(a)} \equiv(-)^{m} c_{m n}^{(r)}, \quad m, n=0, \ldots, N, \\
& c_{m n}^{(e)} \equiv(-)^{m+n} c_{m n}^{(r)}, \quad m, n=0, \ldots, N .
\end{aligned}
$$


The connection of the functions $E_{s}, s=a, e$, to the function $E_{r}$ readily follows from (1.39) and (1.40), resp.: We have

$$
\begin{gathered}
E_{a}(\nu, \beta ; x, p)=(-i)^{N} \exp \left(\frac{\pi p}{2 \hbar \nu}\right) E_{r}\left(\nu, \beta ; x+\frac{i \pi}{2 \nu}, p\right), \\
E_{e}(\nu, \beta ; x, p) \\
=(-)^{N} \exp \left(\frac{\pi x}{2 \hbar \beta}+\frac{\pi p}{2 \hbar \nu}+\frac{i \pi^{2}}{4 \hbar \beta \nu}\right) E_{r}\left(\nu, \beta ; x+\frac{i \pi}{2 \nu}, p+\frac{i \pi}{2 \beta}\right) .
\end{gathered}
$$

The repulsive eigenfunctions were already detailed in Ref. [55] and studied in Ref. [45]. (The dimensionless variable pair $(x, p)$ and parameters $a_{+}, a_{-}, g$ of the latter reference correspond to $(s, t) \equiv(\nu x, \beta p)$ and $\hbar \beta \nu, \pi, g / \hbar$, resp., in the present paper.)

The above eigenfunctions have some crucial symmetry properties, which are equivalent to symmetries of the coefficients $c_{m n}^{(s)}$. Specifically, the repulsive coefficients satisfy (cf. Section II in Ref. [45])

$$
\begin{gathered}
c_{m n}^{(r)}=c_{N-m, N-n}^{(r)}=\bar{c}_{N-m, n}^{(r)}, \\
c_{m n}^{(r)}=c_{n m}^{(r)},
\end{gathered}
$$

so that one has

$$
\begin{gathered}
G_{r}(\nu, \beta ; x, p)=G_{r}(\nu, \beta ;-x,-p)=\overline{G_{r}}(\nu, \beta ;-x, p), \\
G=E, F, \quad x, p \in \mathbb{R}, \\
G_{r}(\nu, \beta ; x, p)=G_{r}(\beta, \nu ; p, x), \quad G=E, F .
\end{gathered}
$$

From (1.39) and (1.40) we then obtain corresponding symmetries of $G_{a}$ and $G_{e}$, and in particular

$$
G_{e}(\nu, \beta ; x, p)=G_{e}(\beta, \nu ; p, x), \quad G=E, F \text {. }
$$

The symmetries (1.46) and (1.47) are particularly striking and useful. Indeed, from these self-duality relations important properties of the adjoint eigenfunction transforms will be immediate.

We continue by sketching the organization of this paper in some detail. In Section 2 we work in a general framework that will be specialized to the above three cases in Sections 3-5. The functional-analytic core of Section 2 (and of the paper) consists of Theorems 2.1-2.3, whose proofs are relegated to Appendix A. 
In Section 2 we work with scaled (dimensionless) variables and make various assumptions that will be shown to be (generically) satisfied in each of the three cases. Actually, we have tried to anticipate its application to a great many special cases of the transforms associated with the generalized hypergeometric function introduced in Ref. [3] (see also Ref. [56]). We will come back to this elsewhere, as well as to the connection of the eigenfunction transforms of Sections 3-5 with the generalized hypergeometric transforms.

Theorem 2.1 paves the way for establishing the isometry properties of the eigenfunction transform. It reveals that an eventual isometry violation is encoded in a non-zero residue operator. The theorem is established without invoking any Hamiltonian. In fact, in Section 2 we need not and do not assume that the transform kernel is an eigenfunction of a non-trivial $\mathrm{A} \Delta \mathrm{O}$. It is, however, manifestly an eigenfunction of a 'free' $\mathrm{A} \Delta \mathrm{O} A_{1}$ (2.18), generalizing the $\mathrm{A} \Delta \mathrm{O} A(1.18)$.

In Theorem 2.2 we show that a non-zero residue operator entails that one cannot interpret the $\mathrm{A} \Delta \mathrm{O} A_{1}$ as a self-adjoint Hilbert space operator (or even a symmetric one), when the action of the latter is defined in the natural way on (a dense subspace of) the range of the generalized eigenfunction transform $\mathcal{F}$ (2.21). The proof applies with obvious changes to any other $\mathrm{A} \Delta \mathrm{O}$ for which the kernel may be an (improper) eigenfunction with real eigenvalues. Thus Theorem 2.2 will enable us to show that self-adjointness (generically) breaks down for $H_{r}, H_{a}$ and $H_{e}$ when $a$ is outside the intervals (1.20) and (1.21), resp.

Assuming a vanishing residue operator, we study in Theorem 2.3 the Hilbert space scattering theory associated with the self-adjoint dynamics $A_{1}$. Though its action is formally free, the scattering is non-trivial. Just as for Theorem 2.2, the proof of Theorem 2.3 applies to a vast class of dynamics, containing in particular the 'interacting' $\mathrm{A} \Delta \mathrm{Os} H_{r}, H_{a}$ and $H_{e}^{2}$ for the relevant specialization. (This is a manifestation of the invariance principle for the wave operators [57].)

It will be clear from the assumptions in Section 2 that the adjoint $\mathcal{F}^{*}$ of the eigenfunction transform $\mathcal{F}$ can be handled along the same lines, using the dual 'free' A $\Delta \mathrm{O} A_{2}(2.19)$ in the role of $A_{1}$ (2.18). We refrain from doing so as regards Theorems 2.2 and 2.3, since we do not need their 'dual counterparts'. We do specify the analog of Theorem 2.1 for $\mathcal{F}^{*}$, however. (Since the repulsive and extra regimes are self-dual, the latter result is needed only in the attractive case.)

The general theory developed in Section 2 enables us to reduce the case analysis in Sections 3-5 to a study of the two pertinent residue operators $R_{2}$ 
(2.32) and $R_{1}$ (2.58). Quite surprisingly, these are finite-rank for arbitrary $a \in(0, \infty)$, and so we need only isolate pertinent linear algebra properties. The algebraic results obtained in Ref. [45] will be crucial in this enterprise.

\section{§2. The General Framework}

As explained above, it is expedient to reduce the bulk of the analysis associated with the above three concrete cases to results obtained in a more general setting. To ease the notation, we use dimensionless variables $(s, t)$ instead of $(x, p)$, and accordingly start from a function of the form

$$
\begin{aligned}
E(s, t) & \equiv e^{\imath s t / a} P\left(e^{s}, e^{t}\right), \quad a \in(0, \infty), \\
P(y, z) & \equiv \sum_{k=0}^{M_{1}} \sum_{l=0}^{M_{2}} a_{k l} y^{M_{1}-2 k} z^{M_{2}-2 l}
\end{aligned}
$$

where $M_{1}, M_{2} \in \mathbb{N}$ and $a_{k l} \in \mathbb{C}$. (This should be compared to (1.34) and (1.35).)

To ensure non-triviality and a convenient normalization, we assume

$$
\left|a_{00}\right|=1
$$

The symmetries (1.43) and their analogs for $c_{m n}^{(a)}$ and $c_{m n}^{(e)}$ are taken into account by assuming

$$
\begin{gathered}
a_{M_{1}-k, M_{2}-l}=\sigma a_{k l}, \quad \sigma \in\{-1,1\}, \\
\bar{a}_{k l}=\sigma a_{M_{1}-k . l}=a_{k, M_{2}-l} .
\end{gathered}
$$

Note that (2.4) and (2.5) amount to

$$
\begin{gathered}
E(-s,-t)=\sigma E(s, t), \\
\bar{E}(s, t)=\sigma E(-s, t)=E(s,-t), \quad s, t \in \mathbb{R} .
\end{gathered}
$$

Obviously, $E(s, t)$ is a joint eigenfunction of the $\mathrm{A} \Delta \mathrm{O}$

$$
B_{1} \equiv(-)^{M_{1}}\left(T_{\imath \pi}^{s}+T_{-i \pi}^{s}\right),
$$

with eigenvalue $2 \operatorname{ch}(\pi t / a)$, and of the dual $\mathrm{A} \Delta \mathrm{O}$

$$
B_{2} \equiv(-)^{M_{2}}\left(T_{\imath \pi}^{t}+T_{-\imath \pi}^{t}\right)
$$

with eigenvalue $2 \operatorname{ch}(\pi s / a)$. Here and below, we find it convenient to encode dependence on the variables $s$ and $t$ by using subscripts 1 and 2, resp. 
Next, we introduce the $c$-functions

$$
c_{1}(s) \equiv \sum_{k=0}^{M_{1}} a_{k 0} e^{\left(M_{1}-2 k\right) s}, \quad c_{2}(t) \equiv \sum_{l=0}^{M_{2}} a_{0 l} e^{\left(M_{2}-2 l\right) t} .
$$

Clearly, $c_{\jmath}(z)$ is an entire function, which is $i \pi$-periodic for $M_{\jmath}$ even and $i \pi$ antiperiodic for $M_{\jmath}$ odd. Using (2.5) one infers

$$
\overline{c_{1}}(s)=\sigma c_{1}(-s), \quad \overline{c_{2}}(t)=c_{2}(-t), \quad s, t \in \mathbb{R},
$$

and using also (2.3) one sees that $c_{\jmath}(z)$ has $M_{\jmath}$ zeros (counting multiplicity) in the period strip $\operatorname{Im} z \in[0, \pi)$. Moreover, from (2.1) and (2.2) one readily deduces

$$
E(s, t)=e^{M I_{2} t} c_{1}(s) e^{2 s t / a}+O\left(\exp \left[\left(M_{2}-2\right) \operatorname{Re} t\right]\right), \quad \operatorname{Re} t \rightarrow \infty
$$

where the bound is uniform for $s$ and $\operatorname{Im} t$ varying over compact subsets of $\mathbb{C}$ and $\mathbb{R}$, resp. Similarly, we have

$$
E(s, t)=e^{M_{1} s} c_{2}(t) e^{i s t / a}+O\left(\exp \left[\left(M_{1}-2\right) \operatorname{Re} s\right]\right), \quad \operatorname{Re} s \rightarrow \infty,
$$

uniformly for $t$ in $\mathbb{C}$-compacts and $\operatorname{Im} s$ in $\mathbb{R}$-compacts.

We proceed by defining weight functions

$$
w_{1}(s) \equiv\left[\sigma c_{1}(s) c_{1}(-s)\right]^{-1}, \quad w_{2}(t) \equiv\left[c_{2}(t) c_{2}(-t)\right]^{-1} .
$$

Thus, $w_{J}(z)$ is an $i \pi$-periodic, meromorphic and even function with $2 I_{J}$ poles (counting multiplicity) for which $\operatorname{Im} z \in[0, \pi)$. Throughout this section we assume that these poles are non-real. Thus they occur at points $p_{1}^{(\jmath)} \ldots, p_{M_{J}}^{(\jmath)}, i \pi-$ $p_{1}^{(\jmath)}, \ldots, i \pi-p_{M I_{J}}^{(\jmath)}$ in the $\operatorname{strip} \operatorname{Im} z \in(0, \pi)$; by convention, the points $p_{k}^{(\jmath)}$ denote the zeros of $c_{\jmath}(-z)$ in this strip. Due to (2.11) and our non-reality assumption, we have

$$
w_{\jmath}(z) \in(0, \infty), \quad \forall z \in \mathbb{R}, \quad j=1,2 .
$$

For later use we also note the asymptotics

$$
w_{\jmath}(z)=e^{-2 M, z}+O\left(e^{\left.-\left(2 M_{1}+2\right) \operatorname{Re} z\right)}\right), \quad \operatorname{Re} z \rightarrow \infty, j=1.2,
$$

where the bounds are uniform in $\operatorname{Im} z$.

We continue by introducing the function

$$
F(s, t)=w_{1}(s)^{1 / 2} E(s, t) w_{2}(t)^{1 / 2} .
$$


As it stands, this function has a sign ambiguity. For real $s$ and $t$ we choose positive square roots throughout, cf. (2.15). Conjugating the A $\Delta \mathrm{Os} B_{1}$ (2.8) and $B_{2}(2.9)$ with the respective weight functions yields

$$
\begin{aligned}
& A_{1}=T_{\imath \pi}^{s}+T_{-\imath \pi}^{s}, \\
& A_{2}=T_{\imath \pi}^{t}+T_{-\imath \pi}^{t} .
\end{aligned}
$$

We are now going to use $F(s, t)$ as the (Schwartz) kernel of a bounded operator $\mathcal{F}$ between Hilbert spaces

$$
\mathcal{H}_{1} \equiv L^{2}(\mathbb{R}, d s), \quad \mathcal{H}_{2} \equiv L^{2}(\mathbb{R}, d t)
$$

To be specific, let us denote the subspace of $\mathcal{H}_{\jmath}$ consisting of $C^{\infty}$-functions whose support is compact and does not include the origin by $\mathcal{C}_{\jmath}, j=1,2$. Then we begin by defining

$$
\mathcal{F}: \mathcal{C}_{2} \rightarrow \mathcal{H}_{1}, \quad \phi(t) \mapsto(2 \pi a)^{-1 / 2} \int_{-\infty}^{\infty} F(s, t) \phi(t) d t .
$$

Though it is clear from the above that the integral is absolutely convergent, it is not immediate that $\mathcal{F}$ maps $\mathcal{C}_{2}$ into $\mathcal{H}_{1}$ and that $\mathcal{F}$ extends to a bounded operator (denoted by the same symbol). It is not hard to see this, however, as we now explain.

Consider first the special case $M_{1}=M_{2}=0$. Then one has

$$
\sigma=1, a_{00} \in\{-1,1\}, \quad w_{1}(z)=w_{2}(z)=1 \quad\left(M_{1}, M_{2}=0\right) .
$$

Hence $\mathcal{F}$ amounts to Fourier transformation, and so $\mathcal{F}$ extends to an isometry from $\mathcal{H}_{2}$ onto $\mathcal{H}_{1}$. In the general case it is therefore clear that $(\mathcal{F} \phi)(s)$ equals the product of $w_{1}(s)^{1 / 2}$ and an entire function of the form

$$
\sum_{k=0}^{M_{1}} e^{\left(M_{1}-2 k\right) s} \hat{\phi}_{k}(s), \quad \hat{\phi}_{k}(s) \in \mathcal{H}_{1},
$$

cf. (2.1) and (2.2). From the bound (2.16) with $j=1$ it then follows that we do have $\mathcal{F} \phi \in \mathcal{H}_{1}$ (recall $w_{1}(s)$ is even).

A slight elaboration on the previous paragraph now shows that $\mathcal{F}$ is bounded: Its kernel can be written as

$$
F(s, t)=\sum_{k=0}^{M_{1}} \sum_{l=0}^{M_{2}} a_{k l}\left(w_{1}(s)^{1 / 2} e^{\left(M_{1}-2 k\right) s}\right) \cdot\left(e^{\imath s t / a}\right) \cdot\left(e^{\left(M_{2}-2 l\right) t} w_{2}(t)^{1 / 2}\right),
$$


and each of the terms in the sum corresponds to the product of three bounded operators.

At this point it is convenient to insert some observations on the adjoint $\mathcal{F}^{*}$ of $\mathcal{F}$, which will be used later on. First, from the boundedness of $\mathcal{F}$ it follows that $\mathcal{F}^{*}$ is defined on all of $\mathcal{H}_{1}$ and bounded as well. Second, one easily verifies that its action is given by

$$
\left(\mathcal{F}^{*} \psi\right)(t)=(2 \pi a)^{-1 / 2} \int_{-\infty}^{\infty} \bar{F}(s, t) \psi(s) d s, \quad \psi \in \mathcal{C}_{1}
$$

Finally, in view of (2.7) we are free to use the equalities

$$
\bar{F}(s, t)=\sigma F(-s, t)=F(s,-t) .
$$

In the following theorem we assume that all poles of $w_{1}(s)$ are simple. The theorem involves a residue function defined by

$$
\begin{aligned}
\mathcal{R}\left(t, t^{\prime}\right) \equiv & \sum_{k=1}^{M_{1}}\left[r_{k}^{(1)} E\left(p_{k}^{(1)},-t\right) E\left(p_{k}^{(1)}, t^{\prime}\right)\right. \\
& \left.+r_{k+M I_{1}}^{(1)} E\left(i \pi-p_{k}^{(1)},-t\right) E\left(i \pi-p_{k}^{(1)}, t^{\prime}\right)\right] .
\end{aligned}
$$

Here, $r_{k}^{(1)}$ and $r_{k+M I_{1}}^{(1)}$ denote the residues of $w_{1}(s)$ at the simple poles $p_{k}^{(1)}$ and $i \pi-p_{k}^{(1)}$, resp. Now $w_{1}(s)$ is $i \pi$-periodic and even, so we have

$$
r_{k+M_{1}}^{(1)}=-r_{k}^{(1)}, \quad k=1, \ldots, M_{1} .
$$

Combining this with (2.6) and (2.1), we deduce

$$
\begin{aligned}
\mathcal{R}\left(t, t^{\prime}\right)= & \sum_{k=1}^{M_{1}} r_{k}^{(1)}\left[E\left(p_{k}^{(1)},-t\right) E\left(p_{k}^{(1)}, t^{\prime}\right)\right. \\
& \left.-\exp \left(\pi\left(t-t^{\prime}\right) / a\right) E\left(p_{k}^{(1)}, t\right) E\left(p_{k}^{(1)},-t^{\prime}\right)\right] .
\end{aligned}
$$

From this one reads off that $\mathcal{R}\left(t, t^{\prime}\right)$ is an entire function satisfying

$$
\mathcal{R}(t, t)=0 .
$$

Theorem 2.1. $\quad$ Assuming $w_{1}(s)$ has solely simple poles, one has

$$
\begin{aligned}
(\mathcal{F} \phi, \mathcal{F} \psi)_{1}= & (\phi, \psi)_{2}+\frac{i}{a} \int_{-\infty}^{\infty} \int_{-\infty}^{\infty} \bar{\phi}(t) \psi\left(t^{\prime}\right) \\
& {\left[1-\exp \left(\pi\left(t-t^{\prime}\right) / a\right)\right]^{-1} \mathcal{R}\left(t, t^{\prime}\right) w_{2}(t)^{1 / 2} w_{2}\left(t^{\prime}\right)^{1 / 2} d t d t^{\prime} }
\end{aligned}
$$

for all $\phi, \psi \in \mathcal{C}_{2}$, with $\mathcal{R}\left(t, t^{\prime}\right)$ given by $(2.29)$. 
The proof of this theorem is relegated to Appendix A. Here we only point out that the vanishing property (2.30) entails that the integral in (2.31) is absolutely convergent and that in Sections 3-5 the assumption of simple poles is satisfied for generic parameters.

Independently of the latter assumption, we may and will define a bounded self-adjoint operator $R_{2}$ by

$$
R_{2} \equiv \mathcal{F}^{*} \mathcal{F}-\mathbf{1}_{2}
$$

We assume from now on that $R_{2}$ has finite rank $L \in \mathbb{N}$. In contrast to the previous requirements we made (which can be readily met), this may seem a rather ad hoc assumption. It is however satisfied for all of the special cases studied below. (In fact, in the concrete settings of Sections $3-5$, the definition (2.32) yields a finite-rank operator $R_{2}$ even when $w_{1}(s)$ has some real poles.)

In order to prepare the ground for later sections we assume once again (until further notice) that $w_{1}(s)$ has ony simple poles. Then it follows from (2.32) that the residue term on the rhs of $(2.31)$ equals $\left(\phi, R_{2} \psi\right)_{2}$. We now consider two important special cases pertaining to the residue sum (2.29): In the first/second case a single term in the sum yields a vanishing/rank-one contribution to $R_{2}$, resp. (The first case is relevant in Sections 4 and 5 , the second one in Sections 3 and 4.)

First, choosing $k \in\left\{1, \ldots, M_{1}\right\}$, suppose that we have an identity

$$
\exp (\pi t / 2 a) E\left(p_{k}^{(1)}, t\right)=\sigma_{k} \exp (-\pi t / 2 a) E\left(p_{k}^{(1)},-t\right), \quad \sigma_{k} \in\{-1,1\} .
$$

Then the corresponding summand in (2.29) clearly vanishes. To be sure, in the present general setting the parity property (2.33) seems very restrictive. Note in particular that in view of (2.1) and (2.2) it is necessary (but by no means sufficient) for (2.33) that $p_{k}^{(1)}$ be of the form

$$
p_{k}^{(1)}=i \pi / 2+i j_{k} a, \quad j_{k} \in \mathbb{Z} .
$$

Second, suppose that $E\left(p_{k}^{(1)}, t\right)$ itself has a definite parity:

$$
E\left(p_{k}^{(1)},-t\right)=\sigma_{k} E\left(p_{k}^{(1)}, t\right), \quad \sigma_{k} \in\{-1,1\} .
$$

Due to (2.1) and (2.2) this implies not only

$$
p_{k}^{(1)}=i j_{k} a, \quad j_{k} \in \mathbb{N}^{*},
$$

but also

$$
\begin{aligned}
E\left(p_{k}^{(1)}, t\right) & =\exp \left[\left(M_{2}+i p_{k}^{(1)} / a\right) t\right] c_{1}\left(p_{k}^{(1)}\right) \\
& +O\left(\exp \left[\left(M_{2}-2+i p_{k}^{(1)} / a\right) t\right]\right), t \rightarrow \infty
\end{aligned}
$$


(Recall that $c_{1}\left(-p_{k}^{(1)}\right)=0$ by convention, so that $c_{1}\left(p_{k}^{(1)}\right) \neq 0$, cf. the paragraph containing (2.14).) Combining (2.16) and (2.37) with (2.35), we deduce

$$
E\left(p_{k}^{(1)}, t\right) w_{2}(t)^{1 / 2} \in \mathcal{H}_{2} .
$$

Moreover, from (2.6), (2.7) and (2.36) we obtain

$$
\bar{E}\left(p_{k}^{(1)}, t\right)=\sigma E\left(p_{k}^{(1)}, t\right), \quad t \in \mathbb{R} .
$$

Therefore, the pertinent summand in (2.31) can be written as

$$
\left.i a^{-1} r_{k}^{(1)} \sigma \sigma_{k}\left(\phi, E\left(p_{k}^{(1)} \cdot\right)\right) w_{2}(\cdot)^{1 / 2}\right)_{2}\left(E\left(p_{k}^{(1)}, \cdot\right) w_{2}(\cdot)^{1 / 2}, \psi\right)_{2} \cdot
$$

As a consequence, it gives rise to a rank-1 operator on $\mathcal{H}_{2}$.

The two cases just considered are not the only way in which the residue sum (2.29) can give rise to a finite-rank operator $R_{2}$, as assumed above. Indeed, we will encounter other possibilities in later sections. But we need not and will not analyze further cases for our remaining purposes in this general section. In fact, for the remainder of this section we drop the assumption that $w_{1}(s)$ has simple poles.

Reconsidering the operator equality (2.32), we observe that the rank- $L$ assumption on $R_{2}$ entails that $R_{2}$ has $L$ non-zero eigenvalues belonging to $[-1,0)$ or $(0, \infty)$. Setting

$$
\mathcal{R}_{2} \equiv R_{2}\left(\mathcal{H}_{2}\right)
$$

it is also clear from (2.32) that $\mathcal{F}$ is isometric on the orthocomplement $\mathcal{R}_{2}^{\perp}$ of the $L$-dimensional range $\mathcal{R}_{2}$.

With these Hilbert space properties at our disposal, we now study the question whether $\mathcal{F}$ may be viewed as an eigenfunction transform for a selfadjoint operator $A_{1}$ associated to the $\mathrm{A} \Delta \mathrm{O}$ (2.18). To analyze this. let us first denote the operator of multiplication by $2 \operatorname{ch}(\pi t / a)$ on $\mathcal{H}_{2}$ by $\mathcal{M}_{2}$, and its natural domain by $\mathcal{D}\left(\mathcal{M}_{2}\right)$. (That is, $\mathcal{D}\left(\mathcal{M}_{2}\right)$ is the maximal multiplication domain, so that $\mathcal{M}_{2}$ is self-adjoint on $\mathcal{D}\left(\mathcal{M}_{2}\right)$.) Now there are two essentially different cases: Either $R_{2}=0$ or $R_{2} \neq 0$.

In the first case $\mathcal{F}$ is an isometry, so $\operatorname{Ker}(\mathcal{F})=0$. Therefore, we may define an operator $A_{1}$ on $\mathcal{F}\left(\mathcal{D}\left(\mathcal{M}_{2}\right)\right)$ by requiring the intertwining relation

$$
A_{1} \mathcal{F}=\mathcal{F M}_{2}
$$

Whenever $\mathcal{F}$ does not map $\mathcal{H}_{2}$ onto $\mathcal{H}_{1}$, this operator is not densely defined, but $A_{1}$ is easily seen to be self-adjoint as an operator on (the subspace $\mathcal{F}\left(\mathcal{D}\left(\mathcal{M}_{2}\right)\right.$ ) of) the Hilbert space 


$$
\mathcal{H}_{1}^{c} \equiv \mathcal{F}\left(\mathcal{H}_{2}\right)
$$

In the second case $\mathcal{F}$ may have a non-zero kernel (cf. (2.32)), so there might exist vectors $\phi$ satisfying

$$
\phi \in \mathcal{D}\left(\mathcal{M}_{2}\right) \cap \operatorname{Ker}(\mathcal{F}), \quad \mathcal{M}_{2} \phi \notin \operatorname{Ker}(\mathcal{F}) .
$$

Whenever such vectors exist, one is not entitled to use (2.42) to define $A_{1}$ on $\mathcal{F}\left(\mathcal{D}\left(\mathcal{M}_{2}\right)\right)$. Now we cannot rule out the obstruction (2.44) in general. On the other hand, when we restrict attention to the smaller ('Paley-Wiener') subspace

$$
\mathcal{P}_{1} \equiv \mathcal{F}\left(\mathcal{C}_{2}\right)
$$

then it can be shown that (2.42) does give rise to a well-defined operator $A_{1}$; stronger yet, one has $\mathcal{C}_{2} \cap \operatorname{Ker}(\mathcal{F})=\{0\}$.

We do not substantiate the latter claim here, since our proof is somewhat involved and since we do not need this result here. Indeed, in the following theorem we only assert that when $R_{2} \neq 0$ and $A_{1}$ can be defined by (2.42) on $\mathcal{P}_{1}$, then the resulting operator is not symmetric.

At this point we would like to emphasize that the action of $A_{1}$ on $\mathcal{P}_{1}$ given by (2.42) is the natural one associated with the $\mathrm{A} \Delta \mathrm{O}$ (2.18): Writing $(\mathcal{F} \phi)(s), \phi \in \mathcal{C}_{2}$, as $w_{1}(s)^{1 / 2}$ times an entire function $\tilde{\phi}(s),(2.42)$ amounts to

$$
\left(A_{1} \mathcal{F} \phi\right)(s) \equiv w_{1}(s-i \pi)^{1 / 2} \tilde{\phi}(s-i \pi)+(i \rightarrow-i) .
$$

as is readily seen from the definition $(2.21)$ of $\mathcal{F}$. It is also important to observe that the intersection of two subspaces $\mathcal{F}\left(\mathcal{C}_{2}\right)$ corresponding to two distinct weight functions $w_{1}$ consists of the zero vector whenever the square roots in (2.46) give rise to branch points at distinct locations. (For example, whenever there exists a point where one of the two weight functions has a simple pole, whereas the other one has a finite value.)

Theorem 2.2. Assuming $R_{2}=0$, the operator $A_{1}$ defined on $\mathcal{P}_{1}$ by (2.42) is essentially self-adjoint (viewed as an operator acting in $\mathcal{H}_{1}^{c}(2.43)$ ). Next, assume $R_{2} \neq 0$ and assume that $\phi \in \mathcal{C}_{2} \cap \operatorname{Ker}(\mathcal{F})$ entails $\mathcal{M}_{2} \phi \in \operatorname{Ker}(\mathcal{F})$. Then the operator $A_{1}$ defined on $\mathcal{P}_{1}$ by $(2.42)$ is not symmetric.

The proof of this theorem can be found in Appendix A. In the remainder of this section we assume $R_{2}=0$. Thus $\mathcal{F}$ is an isometry, and the $\mathrm{A} \Delta \mathrm{O} A_{1}$ (2.18) gives rise to a self-adjoint operator $A_{1}$ acting in the Hilbert space $\mathcal{H}_{1}^{c}$, which is unitarily equivalent to $\mathcal{M}_{2}$ via the intertwining relation (2.42). 
Next, we study the scattering theory associated to the operator $A_{1}$. To this end we extend $A_{1}$ to a self-adjoint operator on $\mathcal{H}_{1}$ by defining $A_{1}$ to be equal to an arbitrary self-adjoint operator on $\left(\mathcal{H}_{1}^{c}\right)^{\perp}$. This extension only serves to let $\exp \left(-i T A_{1}\right), T \in \mathbb{R}$, be a 1-parameter unitary group acting on all of $\mathcal{H}_{1}$; the following scattering theory objects are independent of the extension.

In the present context it is expedient to employ the two Hilbert space scattering theory formalism, cf. Ref. [57]. As comparison operator between the Hilbert spaces $\mathcal{H}_{2}$ and $\mathcal{H}_{1}$ we choose the map

$$
(J \phi)(s) \equiv(2 \pi a)^{-1 / 2} \int_{-\infty}^{\infty} e^{\imath s t / a} \phi(t) d t .
$$

Since $J$ amounts to Fourier transformation, it yields an isometry from $\mathcal{H}_{2}$ onto $\mathcal{H}_{1}$.

Clearly, $J$ intertwines the parity operators

$$
\left(P_{2} \phi\right)(t) \equiv \phi(-t) ; \quad\left(P_{1} \psi\right)(s) \equiv \psi(-s), \quad \phi \in \mathcal{H}_{2}, \quad \psi \in \mathcal{H}_{1}
$$

When $\sigma$ equals 1 , this is true for $\mathcal{F}$ as well, but more generally we have

$$
\mathcal{F} P_{2}=\sigma P_{1} \mathcal{F},
$$

cf. (2.6) and (2.21). Introducing sign functions

$$
\sigma_{-}(t) \equiv\left\{\begin{array}{ll}
\sigma, & t>0 \\
1, & t<0
\end{array}, \quad \sigma_{+}(t) \equiv \sigma \sigma_{-}(t)\right.
$$

we therefore obtain

$$
\mathcal{F} \sigma_{\delta}(t) P_{2}=P_{1} \mathcal{F} \sigma_{\delta}(t), \quad \delta=+,-,
$$

both for $\sigma=1$ and for $\sigma=-1$.

As a last preliminary for the following theorem we recall that $c_{2}(t)$ does not vanish for $t \in \mathbb{R}$ (by assumption, cf. the paragraph containing (2.14)). Moreover, the number $c_{2}(0)$ is real in view of $(2.11)$, so its sign is well defined.

Theorem 2.3. $\quad$ The strong limits of the operator family

$$
\exp \left(i T A_{1}\right) J \exp \left(-i T \mathcal{M}_{2}\right), \quad T \in \mathbb{R},
$$

for $T \rightarrow \pm \infty$ exist and are equal to

$$
W_{ \pm} \equiv \mathcal{F} \sigma_{ \pm}(t)\left[c_{2}(|t|) / c_{2}(-|t|)\right]^{\mp 1 / 2} \operatorname{sign}\left(c_{2}(0)\right) .
$$

Here, the sign functions $\sigma_{ \pm}(t)$ are defined by (2.50), and the square-root signs are chosen such that the resulting one-valued functions are continuous and equal to 1 for $t=0$. 
The proof of Theorem 2.3 is relegated to Appendix A. Just as in nonrelativistic time-independent scattering theory, one can obtain the $S$-matrix

$$
S_{2} \equiv W_{+}^{*} W_{-}=\sigma \frac{c_{2}(|t|)}{c_{2}(-|t|)}
$$

directly from the eigenfunction $F(s, t)$. Indeed, from $(2.17),(2.13),(2.16)$ and (2.6) one infers

$$
F(s, t) \sim \begin{cases}{\left[c_{2}(t) / c_{2}(-t)\right]^{1 / 2} \exp (i s t / a),} & \operatorname{Re} s \rightarrow \infty \\ \sigma\left[c_{2}(-t) / c_{2}(t)\right]^{1 / 2} \exp (i s t / a), & \operatorname{Re} s \rightarrow-\infty\end{cases}
$$

Thus the right and left asymptotics are related via the unitary multiplication operator (2.54). Of course, the latter asymptotics comparison holds true independently of Hilbert space properties. In particular, it fails to reveal that there is no sensible wave packet picture of scattering (indeed, of time evolution) whenever the residue operator $R_{2}$ is non-zero.

The adjoint $\mathcal{F}^{*}(2.25)$ can now be analyzed in the same way as $\mathcal{F}$. However, for later purposes we only need the counterparts of (2.31) and (2.29). (They will be used in Section 4.) An inspection of the proof of Theorem 2.1 in Appendix A readily yields the desired formulas: Provided $w_{2}(t)$ has only simple poles, one gets

$$
\begin{aligned}
\left(\mathcal{F}^{*} \phi, \mathcal{F}^{*} \psi\right)_{2}= & (\phi, \psi)_{1}+\frac{i \sigma}{a} \int_{-\infty}^{\infty} \int_{-\infty}^{\infty} \bar{\phi}(s) \psi\left(s^{\prime}\right) \\
& {\left[1-\exp \left(\pi\left(s^{\prime}-s\right) / a\right)\right]^{-1} \hat{\mathcal{R}}\left(s, s^{\prime}\right) w_{1}(s)^{1 / 2} w_{1}\left(s^{\prime}\right)^{1 / 2} d s d s^{\prime} }
\end{aligned}
$$

for all $\phi, \psi \in \mathcal{C}_{1}$, with

$$
\begin{aligned}
\hat{\mathcal{R}}\left(s, s^{\prime}\right)= & \sum_{l=1}^{M_{2}} r_{l}^{(2)}\left[E\left(-s, p_{l}^{(2)}\right) E\left(s^{\prime}, p_{l}^{(2)}\right)\right. \\
& \left.-\exp \left(\pi\left(s^{\prime}-s\right) / a\right) E\left(s, p_{l}^{(2)}\right) E\left(-s^{\prime}, p_{l}^{(2)}\right)\right] .
\end{aligned}
$$

(The extra factor $\sigma$ in the second term on the rhs of (2.56) as compared to (2.31) arises from using the first equality in (2.7). Recall also (2.14) to see why this factor does not occur in the first term.)

Introducing a second residue operator by setting

$$
R_{1} \equiv \mathcal{F} \mathcal{F}^{*}-\mathbf{1}_{1}
$$

it is straightforward to adapt the remainder of Section 2, with $A_{2}$ playing the role of $A_{1}$. We leave this to the interested reader, however. 


\section{§3. The Repulsive Regime}

Our starting point in this section is the function $E_{r}(x, p)$, given by (1.34)(1.38). (We suppress the parameter dependence whenever this does not give rise to ambiguity.) It follows from Theorem II.1 in Ref. [45] that one has the eigenvalue relation

$$
B_{r} E_{r}(x, p)=2 \operatorname{ch}(\beta p) E_{r}(x, p),
$$

(cf. l.c. Eq. (2.12)), which entails

$$
H_{r} F_{r}(x, p)=2 \operatorname{ch}(\beta p) F_{r}(x, p),
$$

cf. (1.27). Moreover, from this theorem we also have

$$
E_{r}( \pm i N \hbar 3, p)=\prod_{k=N+1}^{2 N} 2 \sin k a
$$

cf. l.c. Eq. (2.16), and l.c. Eq. (2.18) yields

$$
E_{r}( \pm i(N-l) \hbar \beta, p)=B_{l}^{(N)}(\operatorname{ch} 3 p), \quad l=0, \ldots, N,
$$

where $B_{l}^{(N)}(u)$ is a polynomial of degree $\leq l$ and parity $(-)^{l}$ with real coefficients; the degree equals $l$ provided the $a$-restriction

$$
k a \notin \pi \mathbb{N}, \quad k=1 \ldots, 2 N .
$$

is satisfied. Finally, the polynomials obey the recurrence relation

$$
\begin{gathered}
\sin ((2 N-l) a) B_{l+1}^{(N)}(u)-\sin (l a) B_{l-1}^{(N)}(u)=2 u \sin ((N-l) a) B_{l}^{(N)}(u) \\
l=0, \ldots, N
\end{gathered}
$$

(This is simply the eigenvalue formula (3.1), evaluated for the $x$-values in (3.4), cf. (1.27).)

Comparing (1.34) and (1.35) to (2.1) and (2.2), we see that the function

$$
E(s, t) \equiv E_{r}(s / \nu, t / \beta)
$$

is of the form (2.1), with

$$
a=\hbar \beta \nu, \quad M_{1}, M_{2}=N
$$

and coefficients

$$
a_{k \cdot l}=c_{k \cdot l}^{(r)}
$$


In particular, this yields

$$
a_{00}=(-i)^{N} q^{N(N+1) / 2}, \quad q=e^{\imath a},
$$

so that (2.3) is obeyed. Moreover, due to (1.43), the coefficients $a_{k l}$ satisfy (2.4) and (2.5) with the parity parameter $\sigma$ equal to 1 . Accordingly, $E(s, t)$ (3.7) fulfils (2.6) and (2.7) with $\sigma=1$, and in addition

$$
E(s, t)=E(t, s)
$$

since (1.44) entails $a_{k l}=a_{l k}$.

Next, we note that (1.37) yields

$$
c_{m 0}^{(r)}=(-)^{m}(-i)^{N} q^{N(N+1) / 2} \sum_{1 \leq k_{1}<\cdots<k_{m} \leq N} q^{-2\left(k_{1}+\cdots+k_{m}\right)} .
$$

Thus the $c$-function $c_{1}(s)(2.10)$ is given by

$$
\begin{aligned}
c_{1}(s) & =\sum_{i=0}^{N} c_{m 0}^{(r)} e^{(N-2 m) s} \\
& =(-i)^{N} q^{N(N+1) / 2} e^{N \cdot} \prod_{k=1}^{N}\left(1-q^{-2 k} e^{-2 s}\right) \\
& =\prod_{k=1}^{N}(-2 i) \operatorname{sh}(s+i k a),
\end{aligned}
$$

and by symmetry we have $c_{2}(t)=c_{1}(t)$. Recalling (2.14) and (1.25), we now obtain weight functions

$$
u_{1}(s)=w_{s}(s), \quad w_{2}(t)=u_{s}(t) .
$$

It follows from (1.31) and (3.14) that the function

$$
F(s, t) \equiv F_{r}(s / \nu, t / \beta),
$$

is of the form (2.17). Obviously, the weight functions have simple and non-real poles iff the a-restriction (3.5) holds true. This restriction is, in particular, satisfied when

$$
a \in(0, \pi / N), \quad a \neq \frac{\pi}{N+l}, \quad l=1 \ldots, N,
$$

and we now assume (3.16) until further notice. Then we may and will choose

$$
p_{k^{\prime}}^{(J)}=i k a, \quad k=1, \ldots, N, \quad j=1,2 .
$$


(Recall these numbers are by convention the zeros of $c_{\jmath}(-z)$ for $\operatorname{Im} z \in(0, \pi)$.) Moreover, we infer that the assumptions of Theorem 2.1 are met.

From (3.4) and (3.7) we now read off that the second parity assumption (2.35) is satisfied for $k=1, \ldots, N$, with $\sigma_{k}=1$. (Note that the conclusion (2.36) is in accord with (3.17).) Furthermore, these equations entail that the $\mathcal{H}_{2}$-functions $(2.38)$ are given by

$$
\begin{aligned}
\psi_{l}^{(r)}(t) & \equiv B_{l}^{(N)}(\operatorname{ch} t)\left(\prod_{j=1}^{N} 4 \operatorname{sh}(t+i j a) \operatorname{sh}(t-i j a)\right)^{-1 / 2} \\
l & =0, \ldots, N-1
\end{aligned}
$$

Therefore, we obtain an explicit expression for the residue operator $R_{2}(2.32)$ : From (2.31), (2.29) and (2.40) we have

$$
R_{2}=\frac{i}{a} \sum_{l=0}^{N-1} r_{N-l} \psi_{l}^{(r)}(t) \otimes \psi_{l}^{(r)}(t)
$$

with residues given by (cf. (3.14) and (1.25))

$$
\begin{aligned}
r_{m} & =\left[4 i \sin (2 m a) \prod_{k \neq m} 4 \sin (k+m) a \sin (k-m) a\right]^{-1}, \\
m & =1, \ldots, N
\end{aligned}
$$

In view of our standing assumption (3.16), the polynomial $B_{l}^{(N)}$ has degree $l$, and so $R_{2}$ is a rank- $N$ operator.

The upshot is that the operator

$$
\begin{gathered}
\mathcal{F}: \mathcal{H}_{2} \rightarrow \mathcal{H}_{1}, \quad \phi(t) \mapsto(2 \pi a)^{-1 / 2} \int_{-\infty}^{\infty} F(s, t) \phi(t) d t \\
F(s, t)=w_{s}(s)^{1 / 2} E_{r}(s / \nu, t / \beta) w_{s}(t)^{1 / 2}
\end{gathered}
$$

is isometric on $\mathcal{R}_{2}^{\perp}$, but not on the $N$-dimensional subspace $\mathcal{R}_{2}$ (2.41). Now it is clear from (3.19) and (3.18) that $\mathcal{R}_{2}$ belongs to the even subspace $\mathcal{H}_{2,+}$. Since the operator $\mathcal{F}$ intertwines the parity operators (recall (2.51)), it admits restrictions

$$
\mathcal{F}_{\delta}: \mathcal{H}_{2 . \delta} \rightarrow \mathcal{H}_{1, \delta}, \quad \delta=+,- \text {. }
$$

Thus, we are now in the position to deduce that the odd restriction $\mathcal{F}_{-}$is an isometry. 
It is not hard to see that $\mathcal{F}_{-}$is actually onto. Indeed, this follows from an inspection of the adjoint $\mathcal{F}^{*}$. It is given by (2.25). Now from the self-duality relation (3.11) we infer

$$
F(s, t)=F(t, s)
$$

so using (2.26) the adjoint kernel can be rewritten as

$$
\bar{F}(s, t)=F(t,-s) .
$$

Hence we can repeat the above steps for $\mathcal{F}^{*}$, obtaining a residue operator

$$
R_{1}=\frac{i}{a} \sum_{l=0}^{N-1} r_{N-l} \psi_{l}^{(r)}(s) \otimes \psi_{l}^{(r)}(s),
$$

cf. (3.19). This implies that the restriction $\mathcal{F}_{-}^{*}$ to $\mathcal{H}_{1,-}$ is an isometry into $\mathcal{H}_{2 .-}$. Thus we have relations

$$
\mathcal{F}_{-}^{*} \mathcal{F}_{-}=\mathbf{1}_{\mathcal{H}_{2,-},}, \quad \mathcal{F}_{-} \mathcal{F}_{-}^{*}=\mathbf{1}_{\mathcal{H}_{1,-}},
$$

and so bijectivity follows.

When we identify $\mathcal{H}_{1 .-}$ and $\mathcal{H}_{2,-}$ in the obvious way with the Hilbert space

$$
\mathcal{H}_{-} \equiv L_{-}^{2}(\mathbb{R}, d y)
$$

then $\mathcal{F}_{-}$is a skew-adjoint operator with a purely imaginary kernel. Indeed, using (3.25) we may write

$$
\left(\mathcal{F}_{-} \phi\right)(y)=(8 \pi a)^{-1 / 2} \int_{-\infty}^{\infty}\left[F\left(y, y^{\prime}\right)-\bar{F}\left(y^{\prime}, y\right)\right] \phi\left(y^{\prime}\right) d y^{\prime}, \quad \phi \in \mathcal{H}_{-},
$$

and from this formula and (3.24) our assertion is plain. Since $\mathcal{F}_{-}$is also a unitary operator on $\mathcal{H}_{-}$, it now follows that we have

$$
\mathcal{F}_{-}=i\left(P_{+}-P_{-}\right),
$$

where $P_{+}$and $P_{-}$are complementary (orthogonal) projections on $\mathcal{H}_{-}$.

The above results were obtained with the $a$-restriction (3.16) in force. We now summarize our isometry results and extend them to the excluded values in (3.16) in the following theorem.

Theorem 3.1. For all $a \in(0, \pi / N)$ the odd restriction $\mathcal{F}_{-}(3.23)$ of the operator $\mathcal{F}$ given by (3.21) and (3.22) is an isometry onto $\mathcal{H}_{1,-}$. Viewed as an operator on $\mathcal{H}_{-}(3.28), \mathcal{F}_{-}$may be written as (3.30), where the complementary projections $P_{+}$and $P_{-}$are strongly continuous in a for $a \in(0, \pi / N)$. 
Proof. We have already proved the first assertion for $a$ satisfying (3.16). To handle the excluded $a$-values, let us note first of all that for these values the poles of the weight functions are no longer simple, but still non-real. Therefore, the operator $\mathcal{F}(3.21)$ is a well-defined bounded operator for the excluded values, too. Moreover, the factorization (2.24) entails that (3.21) gives rise to a family of bounded operators $\mathcal{F}(a), a \in(0, \pi / N)$, that is strongly continuous in $a$. (Indeed, each of the three operator factors in (2.24) is strongly continuous in $a$ for $a \in(0, \pi / N)$, and the coefficients $a_{k ! l}$ are continuous in $a$ for $a \in(0, \infty)$.)

Recalling now the bijectivity relations $(3.27)$, it follows that $\mathcal{F}_{-}(a)$ is an isometry onto $\mathcal{H}_{1 .-}$ for all $a \in(0, \pi / N)$. Viewing $\mathcal{F}_{-}(a), a \in(0, \pi / N)$, as a strongly continuous family of skew-adjoint unitaries on $\mathcal{H}_{-}(3.28)$, it follows from well-known results (see e.g. Theorem VIII.24 in Ref. [58]) that the spectral projections $P_{+}(a)$ and $P_{-}(a)$ on the eigenvalues $i$ and $-i$ are strongly continuous as well.

Let us now return to the physical variables $x, p$ and parameters $\hbar, \nu, \beta$, fixing $a=\hbar 3 \nu \in(0, \pi / N)$. Then it follows from Theorem 3.1 that the operator

$$
\begin{aligned}
& \mathcal{F}_{r}: L^{2}(\mathbb{R}, d p) \rightarrow L^{2}(\mathbb{R} . d x), \\
& \phi(p) \mapsto(2 \pi \hbar)^{-1 / 2} \int_{-\infty}^{\infty} F_{r}(\nu, \beta: x \cdot p) \phi(p) d p
\end{aligned}
$$

has a restriction $\mathcal{F}_{r,-}$ to the odd subspace $L_{-}^{2}(\mathbb{R}, d p)$ that is an isometry onto $L_{-}^{2}(\mathbb{R}, d x)$. In view of the eigenvalue relation (3.2), the pull-back of the selfadjoint multiplication operator $\phi(p) \mapsto 2 \operatorname{ch}(3 p) \phi(p)$ to $L_{-}^{2}(\mathbb{R}, d x)$ defines a selfadjoint operator $H_{r .-}$ on $L_{-}^{2}(\mathbb{R}, d x)$, whose action on functions $\mathcal{F}_{r .-} \phi$, with $\phi$ an odd $C_{0}^{x}$-function (for example). coincides with that of the $\mathrm{A} \Delta \mathrm{O} H_{r}$ (1.13). Similarly. multiplication by $2 \operatorname{ch}(\pi p / \hbar \nu)$ pulls back to a self-adjoint operator $A_{r .-}$ on $L_{-}^{2}(\mathbb{R}, d x)$, whose action corresponds to the $\mathrm{A} \Delta \mathrm{O} A(1.18)$.

The wave and scattering operators associated with the commuting unitary time evolutions $\exp \left(-i T H_{r ._{-}}\right)$and $\exp \left(-i T A_{r .-_{-}}\right)$can now be read off from Theorem 2.3, cf. the remarks below the proof of Theorem 2.3 in Appendix A. In particular, the $S$-matrix is explicitly given by

$$
S_{r,-}(p)=(-)^{N} \prod_{k=1}^{N} \frac{\operatorname{sh}(\beta|p|+i k a)}{\operatorname{sh}(\beta|p|-i k a)}
$$

cf. (2.54) and (3.13).

Let us next assume that $a$ satisfies (3.16). Then it follows from the above that on the even subspace $L_{+}^{2}(\mathbb{R}, d p)$ the operator $\mathcal{F}_{r .+}$ and its adjoint are not 
isometric. The isometry deviations are encoded in the rank- $N$ operators $R_{2}$ (3.19) and $R_{1}$ (3.26). multiplied by $a$ and with $t \rightarrow \beta p$ and $s \rightarrow \nu x$, resp. For $N>1$ the resulting obstruction to defining the $\mathrm{A} \Delta \mathrm{O} H_{r}$ (1.13) as a selfadjoint operator on $L_{+}^{2}(\mathbb{R}, d x)$ (cf. Theorem 2.2 and the remarks made below its proof in Appendix A) also shows up in a concrete and illuminating way for the $H_{r}$-eigenfunctions $\psi_{i}^{(r)}(\nu x)$, as we now detail.

First, from (3.4) and self-duality we obtain

$$
E_{r}(x, \pm i(N-l) \hbar \nu)=B_{l}^{(N)}(\operatorname{ch} \nu x), \quad l=0, \ldots, N .
$$

Hence (3.2) yields

$$
H_{r} \psi_{l}^{\prime(r)}(\nu x)=2 \cos ((N-l) a) \psi_{l}^{(r)}(\nu x), \quad l=0 \ldots, N-1 .
$$

Now the functions $v_{l}^{(r)}$ satisfy the same recurrence relation as the polynomials $B_{l}^{(N)}$, cf. (3.18). This recurrence is given by (3.6). whence we deduce

$$
\psi_{1}^{(r)}(\nu x)=\cos (N a) \operatorname{ch}(\nu x) \psi_{0}^{(r)}(\nu x)
$$

Moreover. from (3.3) and (3.18) we have

$$
\psi_{0}^{(r)}(\nu x)=\prod_{k=N+1}^{2 N} 2 \sin k a \cdot\left(\prod_{j=1}^{N} 4 \operatorname{sh}(\nu x+i j a) \operatorname{sh}(\nu x-i j a)\right)^{-1 / 2}
$$

From these explicit formulas we read off that the $H_{r}$-eigenfunctions $\dot{\psi}_{0}^{(r)}(\nu x)$ and $\varkappa_{1}^{\prime(r)}(\nu x)$ are either positive or negative for all $x \in \mathbb{R}$ (depending on $a \in(0, \pi / N))$. For $N>1$ they both belong to $L_{+}^{2}(\mathbb{R} . d x)$. so it follows that they are not orthogonal to each other. (We suspect this holds for all pairs $v_{l}^{(r)}(\nu x) \cdot \iota^{\prime}(r)(\nu x), l \neq m$.) Yet, they have distinct real eigenvalues for the $\mathrm{A} \Delta \mathrm{O}$ $H_{r}$ (cf. (3.34)). Clearly; this state of affairs is by itself already an obstruction to reinterpreting $H_{r}$ as a self-adjoint operator on $L_{+}^{2}(\mathbb{R}, d x)$.

It remains to study the eigenfunction transform for the exceptional values in $(0 . \pi / N)$ and for $a \geq \pi / N$. In order to do so, we employ again the variables $s$ and $t$. Also, we first concentrate on the $N=1$ case, since the state of affairs can be made fully explicit for this choice of $N$, and renders the general case more accessible. (Cf. also our recent paper Ref. [53] for further information on the $N=1$ case.)

Accordingly; we focus on the function

$$
E(s, t)=-i e^{\prime s t / a}\left(e^{\imath a}\left[e^{s+t}+e^{-s-t}\right]-e^{-\imath a}\left[e^{s-t}+e^{-s+t}\right]\right),
$$


obtained from (3.7) and (1.34)-(1.38). Fixing first $a \in(0, \pi), a \neq \pi / 2$, the residue operator (3.19) reads

$$
R_{2}=\frac{\sin (2 a)}{a} w_{s}(t)^{1 / 2} \otimes w_{s}(t)^{1 / 2}, \quad w_{s}(t)=1 / 4 \operatorname{sh}(t+i a) \operatorname{sh}(t-i a)
$$

where we used (3.20), (3.18) and (3.3). Now a routine calculation yields

$$
\left(\mathcal{F} w_{s}^{1 / 2}\right)(s)=(\pi / 2 a)^{1 / 2} w_{s}(s)^{1 / 2}
$$

so $R_{2}$ has a non-zero eigenvalue $\pi / 2 a-1$, cf. (2.32).

Turning to the exceptional value $a=\pi / 2$ (cf. (3.16)), we obtain $R_{2}=0$, by continuity in $a$. Thus $\mathcal{F}$ is an isometry onto $L^{2}(\mathbb{R}, d x)$ for $a=\pi / 2$. This can be easily understood from (3.37). Indeed, for $a=\pi / 2$ it reduces to

$$
E(s, t)=4 e^{\imath s t / a} \operatorname{ch} s \operatorname{ch} t, \quad a=\pi / 2,
$$

whereas (3.14) and (1.25) entail

$$
w_{1}(s)=1 / 4 \operatorname{ch}^{2} s, \quad w_{2}(t)=1 / 4 \operatorname{ch}^{2} t, \quad a=\pi / 2 .
$$

Hence (3.22) yields

$$
F(s, t)=e^{\imath s t / a}, \quad a=\pi / 2
$$

so that $\mathcal{F}$ amounts to Fourier transformation.

Likewise we calculate

$$
\begin{aligned}
E(s, t) & =4 i e^{\imath s t / a} \operatorname{sh} s \operatorname{sh} t, \quad a=\pi \\
w_{1}(s) & =1 / 4 \operatorname{sh}^{2} s, \quad w_{2}(t)=1 / 4 \operatorname{sh}^{2} t, \quad a=\pi, \\
F(s, t) & =i e^{\imath s t / a} \operatorname{sign}(s) \operatorname{sign}(t), \quad a=\pi .
\end{aligned}
$$

The double pole at the origin of the weight function entails $w_{s}(t)^{1 / 2} \notin L^{2}(\mathbb{R}, d t)$, so that the rhs of (3.38) is ill defined. But from (3.45) it is plain that $\mathcal{F}$ is an isometry onto $L^{2}(\mathbb{R}, d s)$, so that $R_{2}$ (2.32) vanishes for $a=\pi$. (As a consequence, the spectrum of $R_{2}$ is discontinuous at $a=\pi$.)

Choosing next $a>\pi$, we first dispose of the exceptional values $a=$ $l \pi / 2, l=3,4, \ldots$, cf. (3.5). For $l$ odd, we obtain once more (3.40)-(3.42), up to a sign for $(l-1) / 2$ odd. Similarly, for $l$ even, we reobtain (3.43)-(3.45), up to a sign for $l / 2$ even. Thus $\mathcal{F}$ comes down to Fourier transformation for all of the exceptional $a$-values.

Finally, we study the choice

$$
a \in(n \pi,(n+1) \pi), \quad a \neq(n+1 / 2) \pi, \quad n \in \mathbb{N}^{*} .
$$


Then the zero of $c_{1}(-z)$ for $\operatorname{Im} z \in(0, \pi)$ is given by $i(a-n \pi)$. Using

$$
E(i(a-n \pi), t)=2(-)^{n} \sin (2 a) \exp (n \pi t / a),
$$

we now calculate from (2.29) and (3.20)

$$
\begin{aligned}
\mathcal{R}\left(t, t^{\prime}\right)= & -i \sin (2 a) \exp \left[-n \pi\left(t-t^{\prime}\right) / a\right] \\
& \cdot\left(1-\exp \left[(2 n+1) \pi\left(t-t^{\prime}\right) / a\right]\right) .
\end{aligned}
$$

Substituting this in (2.31), we deduce that $R_{2}(2.32)$ is the finite-rank operator

$$
\begin{aligned}
& R_{2}=\frac{\sin (2 a)}{a} \sum_{l=-n}^{n} f_{-l} \otimes f_{l}, \\
& f_{l}(t) \equiv \exp (l \pi t / a) w_{s}(t)^{1 / 2} .
\end{aligned}
$$

(Note $f_{l} \in \mathcal{H}_{2}$, since $a>n \pi$.)

From the explicit formula (3.49) we now read off that isometry of $\mathcal{F}$ breaks down both on the even and on the odd subspace of $\mathcal{H}_{2}$. (Indeed, the restrictions of $R_{2}$ to $\mathcal{H}_{2,+}$ and $\mathcal{H}_{2,-}$ are clearly rank- $(n+1)$ and rank- $n$ operators, resp.) Accordingly, for the $a$-values (3.46) we cannot associate self-adjoint operators on $L_{\delta}^{2}(\mathbb{R}, d x), \delta=+,-$, to the $\mathrm{A} \Delta \mathrm{O} H_{r}$ (1.13) with $g=2 \hbar$. (More precisely, this cannot be done by exploiting the transforms we have available in this paper.)

Having spelled out the special case $N=1$, we supply less detail for the general $N$ case. Consider first the excluded $a$-values in (3.16), corresponding to the presence of double poles in the weight function $w_{s}(y)(1.25)$. It is by no means obvious, but true that all of the double pole factors are matched by similar factors in $E(s, t)$. As a consequence, the function $F(s, t)(3.22)$ reduces to a function of the same type, but with a smaller value of $N$. Making the $N$ and $a$-dependence explicit, this reduction can be specified as

$$
F_{N}\left(\frac{\pi}{N+l} ; s, t\right)=F_{l-1}\left(\frac{\pi}{N+l} ; s, t\right), \quad l=1, \ldots, N .
$$

Thus $\mathcal{F}$ reduces to Fourier transformation for $l=1$, as we have already seen for $N=1$, cf. (3.42). For $l>1$ we have $a<\pi / 2 l$, so that $\mathcal{F}_{-}$is isometric and $R_{2}$ is a rank- $(l-1)$ operator.

The reduction just detailed can be easily derived from Eq. (2.100) in Ref. [45]. In this connection we also point out that we have

$$
F_{N}(a ; s, t)=i F(a, \pi,(N+1) a ; s, t),
$$


where the function on the rhs is defined in Section 3 of Ref. [45], cf. in particular l.c. Eq. (3.41). (The above reduction may be viewed as a manifestation of the $\left(b \rightarrow a_{+}+a_{-}-b\right)$-symmetry of the function $F\left(a_{+}, a_{-}, b ; s, t\right)$, cf. l.c. Eq. (3.52).)

More generally, a similar decrease of $N$ occurs for the excluded values in (3.5). Specifically, assume that $a$ is of the form

$$
\begin{gathered}
a=\pi l / k=\pi s / r, \quad l \in \mathbb{N}^{*}, \\
k \in\{1, \ldots, 2 N\}, \quad s, r \in \mathbb{N}^{*}, \quad s, r \text { coprime. }
\end{gathered}
$$

Then Theorem II.3 in Ref. [45] applies. It entails that all of the functions $F_{L}(\pi s / r ; s, t), L \in \mathbb{N}^{*}$, reduce to one of the functions $F_{n}(\pi s / r ; s, t), n \in$ $\left\{0.1, \ldots, L_{r}\right\}$, with

$$
L_{r} \equiv\left\{\begin{array}{l}
r / 2-1, \quad r \text { even } \\
r / 2-1 / 2, r \text { odd }
\end{array}\right.
$$

For these $n$-values one has $2 n<r$, so that the restriction (3.5) with $N \rightarrow n$ is fulfilled. (To be quite precise, the reduction holds true up to phases in $\{ \pm 1, \pm i\}$ and sign functions; cf. the above special case $N=1$ to see what is involved.)

As a consequence, we are reduced to studying what happens for $a>\pi / N$, with the $a$-restriction (3.5) in effect. The zeros of $c_{1}(-z)$ in the strip $\operatorname{Im} z \in$ $(0, \pi)$ are then given by

$$
p_{k}^{(1)}=i k a-i n_{k} \pi, \quad k=1, \ldots, N,
$$

with $0 \leq n_{1} \leq \cdots \leq n_{N}$ and $n_{N}>0$. Thus we obtain

$$
E\left(p_{k}^{(1)}, t\right)=(-)^{n_{k}} \exp \left(\pi n_{k} t / a\right) B_{N-k}^{(N)}(\operatorname{ch} t) .
$$

From this we deduce just as in the $N=1$ case that $R_{2}$ is given by

$$
\begin{gathered}
R_{2}=\frac{i}{a} \sum_{k=1}^{N} r_{k} \sum_{l=-n_{h}}^{n_{h}} f_{k,-l}^{(r)}(t) \otimes f_{k l}^{(r)}(t), \\
f_{k \cdot l}^{(r)}(t) \equiv \exp (l \pi t / a) \psi_{N-k}^{(r)}(t) .
\end{gathered}
$$

It is not hard to see that the functions $f_{k l}^{(r)}(t)$ give rise to linearly independent vectors in the Hilbert space $\mathcal{H}_{2}$. Also. as $a$ increases, the number $n_{k}$ increases by one whenever $a$ passes a number in $\mathbb{N}^{*} \pi / k$. Correspondingly, we introduce

$$
\kappa_{N}(a) \equiv \sum_{k=1}^{N} \operatorname{card}\left\{n \in \mathbb{N}^{*} \mid n \pi<k a\right\}
$$


where 'card' stands for 'cardinality'. Then a moment's thought shows that one has

$$
\begin{gathered}
\operatorname{rank}\left(R_{2,+}\right)=N+\kappa_{N}(a), \\
\operatorname{rank}\left(R_{2,-}\right)=\kappa_{N}(a),
\end{gathered}
$$

where $R_{2 . \delta}$ denote the restrictions of $R_{2}$ to $\mathcal{H}_{2, \delta}, \delta=+,-$.

Let us now summarize the above analysis. We have established that the ranks of the even and odd parts of $R_{2}$ are increasing functions of $a$ on the subset of $(0, \infty)$ defined by the $a$-restriction (3.5). Both ranks are generically non-zero for $a>\pi / N$, entailing violation of isometry and self-adjointness. For the discrete set of critical $a$-values, the ranks jump down to integers of the form $l+\kappa_{l}(a), \kappa_{l}(a)$, with $l<N$. In particular, when $(N+1) a$ is a multiple of $\pi$, one readily verifies that $l=0$, and that $\mathcal{F}$ amounts to Fourier transformation. This is in accordance with the Hamiltonian $H_{r}$ (1.13) becoming formally 'free' when $g \beta \nu=(N+1) a$ equals $k \pi, k \in \mathbb{N}^{*}$. Observe, however, that the eigenfunction transform $\mathcal{F}_{r}(3.31)$ is not periodic in $a$, in contrast to the $\mathrm{A} \Delta \mathrm{O} H_{r}$.

\section{§4. The Attractive Regime}

The operator $B_{a}(1.28)$ arises from $B_{r}(1.27)$ by the crossing substitution $x \rightarrow x+i \pi / 2 \nu$. Thus the eigenvalue equation (3.1) and the relation (1.41) between $E_{a}$ and $E_{r}$ entail

$$
B_{a} E_{a}(x, p)=2 \operatorname{ch}(\beta p) E_{a}(x, p)
$$

From (1.32) and (1.28) we then deduce

$$
H_{a} F_{a}(x, p)=2 \operatorname{ch}(\beta p) F_{a}(x, p) .
$$

Next, we combine (3.3) and (3.4) with (1.41) to obtain

$$
\begin{gathered}
E_{a}\left(\frac{i \pi}{2 \nu}+i N \hbar \beta, p\right)=i^{N} \exp \left(-\frac{\pi p}{2 \hbar \nu}\right) \prod_{k=N+1}^{2 N} 2 \sin k a \\
E_{a}\left(\frac{i \pi}{2 \nu}+i(N-l) \hbar \beta, p\right)=i^{N} \exp \left(-\frac{\pi p}{2 \hbar \nu}\right) B_{l}^{(N)}(\operatorname{ch} \beta p), l=0, \ldots, N .
\end{gathered}
$$

From (1.41) and the self-duality relations (1.46) we also infer that $B_{a}$ has eigenfunctions

$$
E_{a}(x, i(N-l) \hbar \nu)=(-i)^{l} B_{l}^{(N)}(i \operatorname{sh} \nu x), \quad l=0, \ldots, N,
$$


with eigenvalues $2 \cos (N-l) a$, cf. (4.1).

Just as in the repulsive case, the function

$$
E(s, t) \equiv E_{a}(s / \nu, t / \beta)
$$

amounts to (2.1) with $a=\hbar \beta \nu, M_{1}, M_{2}=N$, and $a_{k l}=c_{k l}^{(a)}$. From (1.39) and (1.43) we have

$$
c_{m n}^{(a)}=(-)^{N} c_{N-m, N-n}^{(a)}=(-)^{N} \bar{c}_{N-m, n}^{(a)},
$$

so that the assumptions (2.4) and (2.5) are satisfied with

$$
\sigma=(-)^{N}
$$

Thus $E(s, t)(4.6)$ satisfies (2.6) and (2.7) with $\sigma=(-)^{N}$.

Proceeding as in the repulsive case (cf. (3.13)), we obtain $c$-functions

$$
c_{1}(s)=\prod_{k=1}^{N}(-2 i) \operatorname{ch}(s+i k a), \quad c_{2}(t)=\prod_{l=1}^{N}(-2 i) \operatorname{sh}(t+i l a) .
$$

Thus we get weight functions

$$
w_{1}(s)=w_{c}(s), \quad w_{2}(t)=w_{s}(t)
$$

so that

$$
F(s, t) \equiv F_{a}(s / \nu, t / \beta)
$$

is of the form (2.17), cf. (1.32). As in the repulsive case, the weight functions have simple and non-real poles iff (3.5) is obeyed, and in particular this is the case for

$$
a \in(0, \pi / 2 N)
$$

Assuming (4.12) till further notice, we may and will take

$$
p_{k}^{(1)}=\frac{i \pi}{2}+i k a, \quad p_{k}^{(2)}=i k a, \quad k=1, \ldots, N
$$

and Theorem 2.1 applies. In view of (4.4), the first parity assumption (2.33) is satisfied with $\sigma_{k}=1, k=1, \ldots, N$. Thus we obtain

$$
R_{2}=0
$$

and so the eigenfunction transform $\mathcal{F}(2.21)$ is an isometry. 
Next, we use the counterpart (2.56) of (2.31) to study the adjoint $\mathcal{F}^{*}$. From (4.5) and (4.13) we see that the (counterpart of the) second parity assumption (2.35) is met with $\sigma_{k}=(-)^{N-k}$. (Recall $B_{l}^{(N)}(u)$ has parity $(-)^{l}$, cf. the recurrence relation (3.6).) From (2.56)-(2.58) we now deduce

$$
R_{1}=\frac{i}{a} \sum_{l=0}^{N-1}(-)^{N+l} r_{N-l} \psi_{l}^{(a)}(s) \otimes \psi_{l}^{(a)}(s),
$$

where the residues are once more given by (3.20) and where

$$
\begin{aligned}
& \psi_{l}^{(a)}(s)=(-i)^{l} B_{l}^{(N)}(i \operatorname{sh} s)\left(\prod_{j=1}^{N} 4 \operatorname{ch}(s+i j a) \operatorname{ch}(s-i j a)\right)^{-1 / 2} \\
& l=0, \ldots, N-1 .
\end{aligned}
$$

(Observe that $\psi_{l}^{(a)}(s)$ is real-valued for $s \in \mathbb{R}$.)

Now we have already shown that $\mathcal{F}$ is isometric. Therefore we have

$$
\mathcal{F F}^{*}=\mathbf{1}_{1}-P_{1},
$$

where $P_{1}$ is the projection on $\left(\mathcal{H}_{1}^{c}\right)^{\perp}$. (Recall $\mathcal{H}_{1}^{c}$ is the range of $\mathcal{F}$, cf. (2.43).) Comparing (4.17) and (2.58), we infer

$$
P_{1}=-R_{1} \text {. }
$$

As a consequence, the subspace $\left(\mathcal{H}_{1}^{c}\right)^{\perp}$ of $\mathcal{H}_{1}$ is $N$-dimensional and spanned by the functions $\psi_{l}^{(a)}(s), l=0, \ldots, N-1$. In the following theorem we summarize the results just obtained and prove moreover that the latter functions are pairwise orthogonal.

Theorem 4.1. For all $a \in(0, \pi / 2 N)$ the operator

$$
(\mathcal{F} \phi)(s)=(2 \pi a)^{-1 / 2} \int_{-\infty}^{\infty} w_{c}(s)^{1 / 2} E_{a}(s / \nu, t / \beta) w_{s}(t)^{1 / 2} \phi(t) d t, \quad \phi \in \mathcal{H}_{2},
$$

is an isometry. The orthocomplement of its range is spanned by the functions $\psi_{l}^{(a)}(s)$ (4.16). The latter satisfy

$$
\left(\psi_{l}^{(a)}(\cdot), \psi_{m}^{(a)}(\cdot)\right)_{1}=\delta_{l m} \mu_{l}^{-1}, \quad l, m=0, \ldots, N-1,
$$

with $\mu_{l}$ defined by

$$
\begin{gathered}
\mu_{l} \equiv\left[4 a \sin (2(N-l) a) \prod_{\substack{k=1 \\
k \neq N-l}}^{N} 4 \sin ((N-l+k) a)|\sin ((N-l-k) a)|\right]^{-1} \\
\quad l=0, \ldots, N-1 .
\end{gathered}
$$


Proof. We have already derived the first two results from Theorem 2.1. To obtain (4.20), we first show that $\psi_{l}^{(a)}$ is orthogonal to $\psi_{m}^{(a)}$ for $l \neq m$. This is equivalent to $b_{l} \perp b_{m}$ in the Hilbert space $L^{2}\left(\mathbb{R}, w_{c}(s) d s\right)$, with

$$
b_{l}(s) \equiv B_{l}^{(N)}(i \operatorname{sh} s) .
$$

To prove $b_{l} \perp b_{m}$, consider the integral

$$
\begin{gathered}
I_{l m} \equiv \int_{-\infty}^{\infty}\left(\frac{\operatorname{ch}(s-i N a)}{\operatorname{ch} s} b_{l}(s+i a)+(i \rightarrow-i)\right) \\
b_{m}(s) \prod_{j=1}^{N}[4 \operatorname{ch}(s+i j a) \operatorname{ch}(s-i j a)]^{-1} d s,
\end{gathered}
$$

with $a \in(0, \pi / 2 N)$. After cancellation of $\operatorname{ch}(s \pm i N a)$, it is plain that we are free to shift contours $s \rightarrow s-i a$ in the first contribution to $I_{l m}$ and $s \rightarrow s+i a$ in the second one, yielding

$$
\begin{aligned}
I_{l m}= & \int_{-\infty}^{\infty} b_{l}(s)\left(\frac{\operatorname{ch}(s+i N a)}{\operatorname{ch} s} b_{m}(s-i a)+(i \rightarrow-i)\right) \\
& \prod_{J=1}^{N}[4 \operatorname{ch}(s+i j a) \operatorname{ch}(s-i j a)]^{-1} d s .
\end{aligned}
$$

Recalling (1.28), (4.1) and (4.5), we now conclude that the right-hand sides of (4.23) and (4.24) equal $2 \cos ((N-l) a)\left(b_{l}, b_{m}\right)$ and $2 \cos ((N-m) a)\left(b_{l}, b_{m}\right)$, resp. Since $a \in(0, \pi / 2 N)$, the numbers up front are distinct, so we must have $\left(b_{l}, b_{m}\right)=0$.

It remains to prove (4.20) for $l=m$. Since we have already shown pairwise orthogonality for $l \neq m$, this norm formula can be easily deduced from (4.15), (3.20) and (4.18).

Returning to the physical variables $x, p$ and parameters $\hbar, \nu, \beta$, we now fix $a=\hbar \beta \nu \in(0, \pi / 2 N)$. Then Theorem 4.1 entails that the operator

$$
\begin{gathered}
\mathcal{F}_{a}: L^{2}(\mathbb{R}, d p) \rightarrow L^{2}(\mathbb{R}, d x), \\
\phi(p) \mapsto(2 \pi \hbar)^{-1 / 2} \int_{-\infty}^{\infty} F_{a}(\nu, \beta ; x, p) \phi(p) d p
\end{gathered}
$$

is an isometry. Due to (4.2), multiplication by $2 \operatorname{ch} \beta p$ pulls back to a self-adjoint operator $H_{a}$ on the range of $\mathcal{F}_{a}$, whose action on $\mathcal{F}_{a} \phi, \phi \in C_{0}^{\infty}$, coincides with the action of the $\mathrm{A} \Delta \mathrm{O}$ (1.14). The orthocomplement of the range is spanned by the orthonormal real-valued functions 


$$
b_{l}^{(N)}(x) \equiv\left(\nu \mu_{l}\right)^{1 / 2}(-i)^{l} B_{l}^{(N)}(i \operatorname{sh} \nu x) \prod_{j=1}^{N}[4 \operatorname{ch}(\nu x+i j a) \operatorname{ch}(\nu x-i j a)]^{-1 / 2},
$$

which are eigenfunctions of the $\mathrm{A} \Delta \mathrm{O}$ (1.14) with eigenvalues $2 \cos (N-l) a, l=$ $0, \ldots, N-1$. Thus we may and will extend the Hilbert space operator $H_{a}$ associated to (1.14) in the obvious way to a self-adjoint operator on $L^{2}(\mathbb{R}, d x)$. This operator has absolutely continuous spectrum $[2, \infty)$ with multiplicity two, and a non-degenerate bound state spectrum

$$
\sigma_{p . p .}=\{2 \cos a, 2 \cos 2 a, \ldots, 2 \cos N a\} .
$$

Likewise, the self-adjoint multiplication operator $\phi(p) \mapsto 2 \operatorname{ch}(\pi p / \hbar \nu) \phi(p)$ gives rise to a self-adjoint operator $A_{a}$ on the range of $\mathcal{F}$ whose action equals that of the $\mathrm{A} \Delta \mathrm{O}$ (1.18). The latter has eigenvalues $2(-)^{N-l}$ on the functions $b_{l}^{(N)}(x)$, and so we define $A_{a}$ in the same way.

The wave and scattering operators associated to the evolutions $\exp \left(-i T H_{a}\right)$ and $\exp \left(-i T A_{a}\right)$ are readily obtained from Theorem 2.3. Specifically, from (2.54) and (4.9) we deduce

$$
S_{2}(p)=\prod_{k=1}^{N} \frac{\operatorname{sh}(\beta|p|+i k a)}{\operatorname{sh}(\beta|p|-i k a)}
$$

Observe that this attractive $S$-matrix differs by a sign from the repulsive one (3.32) when $N$ is odd.

We conclude this section by studying the eigenfunction transform for $a \geq$ $\pi / 2 N$, using once more the dimensionless variables $s$ and $t$. Following Section 3 , we begin with the special case $N=1$, where we have

$$
E(s, t)=-i e^{\imath s t / a}\left(e^{\imath a}\left[e^{s+t}-e^{-s-t}\right]-e^{-\imath a}\left[e^{s-t}-e^{-s+t}\right]\right) .
$$

For the exceptional values $a=k \pi / 2, k \in \mathbb{N}^{*}$, we calculate

$$
\begin{aligned}
& E(s, t)=\left\{\begin{array}{l}
4(-)^{n} e^{\imath s t / a} \operatorname{sh} s \operatorname{ch} t, \quad a=(n+1 / 2) \pi, \\
4 i(-)^{n+1} e^{\imath s t / a} \operatorname{ch} s \operatorname{sh} t, a=n \pi,
\end{array}\right. \\
& w_{1}(s)=\left\{\begin{array}{ll}
1 / 4 \operatorname{sh}^{2} s, \\
1 / 4 \operatorname{ch}^{2} s,
\end{array} \quad w_{2}(t)= \begin{cases}1 / 4 \operatorname{ch}^{2} t, & a=(n+1 / 2) \pi, \\
1 / 4 \operatorname{sh}^{2} t, & a=n \pi\end{cases} \right. \\
& F(s, t)= \begin{cases}(-)^{n} e^{\imath s t / a} \operatorname{sign}(s), & a=(n+1 / 2) \pi, \\
(-)^{n+1} i e^{\imath s t / a} \operatorname{sign}(t), & a=n \pi .\end{cases}
\end{aligned}
$$


Thus $\mathcal{F}$ boils down to Fourier transformation, entailing $R_{2}=R_{1}=0$.

Next, we choose

$$
a \in((n-1 / 2) \pi,(n+1 / 2) \pi), \quad a \neq n \pi, \quad n \in \mathbb{N}^{*} .
$$

Then the zero of $c_{1}(-z)$ for $\operatorname{Im} z \in(0, \pi)$ equals $i a-i(n-1 / 2) \pi$. Now we have

$$
E(i a-i(n-1 / 2) \pi, t)=2 i(-)^{n} \sin (2 a) \exp [(n-1 / 2) \pi t / a],
$$

so (2.29) becomes

$$
\begin{aligned}
\mathcal{R}\left(t, t^{\prime}\right)= & -i \sin (2 a) \exp \left[-(n-1 / 2) \pi\left(t-t^{\prime}\right) / a\right] \\
& \cdot\left(1-\exp \left[2 n \pi\left(t-t^{\prime}\right) / a\right]\right) .
\end{aligned}
$$

From (2.31) we then obtain

$$
R_{2}=\frac{\sin (2 a)}{a} \sum_{l=-n+1 / 2}^{n-1 / 2} f_{-l} \otimes f_{l},
$$

with $f_{l}$ given by (3.50).

As a result, the even and odd restrictions of $R_{2}$ are rank- $n$ operators. Therefore isometry of $\mathcal{F}$ breaks down and we cannot associate self-adjoint operators to the $\mathrm{A} \Delta \mathrm{O} H_{a}$ (1.14) for $g=2 \hbar$ and the $a$-values (4.33). (Just as in Section 3, we should add the qualifier that this is not feasible with the transforms at our disposal in this paper.)

The $N>1$ case can now be studied along the same lines as in the repulsive regime. The point is that the reduction phenomenon detailed there is basically the same for the attractive regime, as we have already seen explicitly for $N=1$. This is because the pertinent functions are related by an $(s \rightarrow s+i \pi / 2)$ continuation, cf. (1.32) and (1.41). Therefore we need only study $R_{2}$ for $a$ values larger than $\pi / 2 N$, with the restriction (3.5) in force.

Accordingly, we begin by noting that the pertinent zeros of $c_{1}(-z)$ read

$$
p_{k}^{(1)}=\frac{i \pi}{2}+i k a-i n_{k} \pi, \quad k=1, \ldots, N
$$

This entails

$$
E\left(p_{k}^{(1)}, t\right)=(-)^{n_{k}} \exp \left[\pi\left(n_{k}-1 / 2\right) t / a\right] B_{N-k}^{(N)}(\operatorname{ch} t),
$$

whence we deduce as before

$$
R_{2}=\frac{i}{a} \sum_{k=1}^{N} r_{k} \sum_{l=-n_{k}+1 / 2}^{n_{k}-1 / 2} f_{k \cdot-l}^{(r)}(t) \otimes f_{k l}^{(r)}(t),
$$


with $f_{k l}^{(r)}$ given by (3.58). (When $n_{k}=0$, the $k$ th term is understood to be absent, of course.)

In the present case the number $n_{k}$ increases by one whenever $a$ passes a number in $\left(\mathbb{N}^{*}-1 / 2\right) \pi / k$, cf. (4.37). Introducing

$$
\nu_{N}(a) \equiv \sum_{k=1}^{N} \operatorname{card}\left\{n \in \mathbb{N}^{*} \mid(n-1 / 2) \pi<k a\right\}
$$

we conclude

$$
\operatorname{rank}\left(R_{2, \delta}\right)=\nu_{N}(a), \quad \delta=+,-
$$

(This should be compared to (3.59)-(3.61).) In particular, the ranks are generically non-zero for $a>\pi / 2 N$, so that isometry and self-adjointness break down. Just as in Section 3, the ranks jump down to $\nu_{l}(a)$ with $l<N$ whenever $a$ takes on one of its critical values, with multiples of $\pi(N+1)$ yielding $l=0$ and $\mathcal{F}_{a}$ (4.25) becoming essentially Fourier transformation.

\section{§5. The Extra Regime}

Using the eigenvalue formula (3.1) and the relation (1.42) between $E_{e}$ and $E_{r}$, one readily verifies that the $\mathrm{A} \Delta \mathrm{O} B_{e}(1.29)$ satisfies

$$
B_{e} E_{e}(x, p)=2 \operatorname{sh}(\beta p) E_{e}(x, p)
$$

Then (1.33) and (1.29) yield

$$
H_{e} F_{e}(x, p)=2 \operatorname{sh}(\beta p) F_{e}(x, p) .
$$

Combining (3.4) and (1.42), we also deduce

$$
\begin{gathered}
E_{e}\left(\frac{i \pi}{2 \nu}+i(N-l) \hbar \beta, p\right)=i^{N-l} \exp \left(-\frac{\pi p}{2 \hbar \nu}\right) B_{l}^{(N)}(i \operatorname{sh} \beta p) \\
l=0, \ldots, N
\end{gathered}
$$

Moreover, the function

$$
E(s, t) \equiv E_{e}(s / \nu, t / \beta)
$$

is of the form (2.1), with $a=\hbar \beta \nu, M_{1}, M_{2}=N$, and $a_{k l}=c_{k l}^{(e)}$. Then we have $\sigma=1$ in (2.4) and (2.5), so $E(s, t)$ satisfies the assumptions (2.6) and (2.7) with $\sigma=1$. Moreover, the self-duality relation (3.11) holds true. 
Proceeding as before, we calculate the $c$-functions

$$
c_{1}(s)=c_{2}(s)=\prod_{k=1}^{N}(-2 i) \operatorname{ch}(s+i k a),
$$

yielding weight functions

$$
w_{1}(s)=w_{2}(s)=w_{c}(s)
$$

Therefore, the function

$$
F(s, t) \equiv F_{e}(s / \nu, t / \beta)
$$

with $F_{e}$ given by (1.33), is of the form (2.17). Imposing the $a$-restriction (4.12) until further notice, the weight functions have simple and non-real poles. Then we may and will choose

$$
p_{k}^{(\jmath)}=\frac{i \pi}{2}+i k a, \quad k=1, \ldots, N, \quad j=1,2 .
$$

We are now in the position to invoke Theorem 2.1. From (5.3) we see that the parity assumption (2.33) holds true for $k=1, \ldots, N$, with $\sigma_{k}=(-)^{N-k}$. Hence we deduce $R_{2}=0$ and isometry of $\mathcal{F}$. By virtue of self-duality, we also have $R_{1}=0$, and so $\mathcal{F}$ maps onto $\mathcal{H}_{1}$.

Identifying $\mathcal{H}_{1}$ and $\mathcal{H}_{2}$ with

$$
\mathcal{H} \equiv L^{2}(\mathbb{R}, d y)
$$

we may view $\mathcal{F}$ as a unitary operator on $\mathcal{H}$. The kernel function $F\left(y, y^{\prime}\right)$ is symmetric (by self-duality), and so (2.26) with $\sigma=1$ entails

$$
\left(\mathcal{F}_{ \pm} \phi\right)(y)=(8 \pi a)^{-1 / 2} \int_{-\infty}^{\infty}\left[F\left(y, y^{\prime}\right) \pm \bar{F}\left(y^{\prime}, y\right)\right] \phi\left(y^{\prime}\right) d y^{\prime}, \quad \phi \in \mathcal{H}_{ \pm}
$$

We are now prepared for the following theorem.

Theorem 5.1. For all $a \in(0, \pi / 2 N)$ the operator $\mathcal{F}$ on $\mathcal{H}$ is unitary. Its restrictions to the even and odd subspaces $\mathcal{H}_{\delta}, \delta=+,-$, satisfy

$$
\begin{aligned}
& \mathcal{F}_{+}=P_{+}^{(+)}-P_{-}^{(+)}, \\
& \mathcal{F}_{-}=i\left(P_{+}^{(-)}-P_{-}^{(-)}\right) .
\end{aligned}
$$

Here, $P_{+}^{(\delta)}$ and $P_{-}^{(\delta)}$ are complementary orthogonal projections on $\mathcal{H}_{\delta}$ that are strongly continuous in a for $a \in(0, \pi / 2 N), \delta=+,-$. 
Proof. We already proved unitarity. From (5.10) we deduce that $\mathcal{F}_{+}$ $\left(\mathcal{F}_{-}\right)$is a unitary self-adjoint (skew-adjoint) operator on $\mathcal{H}_{+}\left(\mathcal{H}_{-}\right)$. Hence the remaining assertions readily follow, cf. also the proof of Theorem 3.1.

Fixing $a \in(0, \pi / 2 N)$, it follows from the above that the operator

$$
\begin{aligned}
\mathcal{F}_{e}: L^{2}(\mathbb{R}, d p) \rightarrow L^{2}(\mathbb{R}, d x), \\
\phi(p) \mapsto(2 \pi \hbar)^{-1 / 2} \int_{-\infty}^{\infty} F_{e}(\nu, \beta ; x, p) \phi(p) d p
\end{aligned}
$$

is an isometric isomorphism. It pulls back the multiplication by $2 \operatorname{sh}(\beta p)$ and $2 \operatorname{ch}(\pi p / \hbar \nu)$ to self-adjoint operators $H_{e}$ and $A_{e}$ on $L^{2}(\mathbb{R}, d x)$, whose actions are given by the $\mathrm{A} \Delta \mathrm{Os}$ (1.15) and (1.18), resp.

In the present case the wave and scattering operators associated to the commuting unitary time evolutions $\exp \left(-i T H_{e}\right)$ and $\exp \left(-i T A_{e}\right)$ are no longer equal. For the latter evolution Theorem 2.3 applies verbatim, yielding the $S$ matrix

$$
S_{2}(p)=\prod_{k=1}^{N} \frac{\operatorname{ch}(\beta|p|+i k a)}{\operatorname{ch}(\beta|p|-i k a)},
$$

cf. (2.54) and (5.5). But for the former we need the modification of Theorem 2.3 detailed at the end of Appendix A, cf. (A.49)-(A.52). In particular, the $S$ matrix corresponding to $\exp \left(-i T H_{e}\right)$ reads

$$
\tilde{S}_{2}(p)=\prod_{k=1}^{N} \frac{\operatorname{ch}(\beta p+i k a)}{\operatorname{ch}(\beta p-i k a)},
$$

cf. (A.52).

As before, our last topic in this section concerns the operator $\mathcal{F}$ for $a \in$ $[\pi / 2 N, \infty)$, using once more the variables $s$ and $t$, and following our account in the two previous sections. Thus we first consider

$$
E(s, t)=-i e^{\imath s t / a}\left(e^{\imath a}\left[e^{s+t}+e^{-s-t}\right]+e^{-i a}\left[e^{s-t}+e^{-s+t}\right]\right) .
$$

As the analogs of (4.30)-(4.32) we then get

$$
\begin{aligned}
& E(s, t)=\left\{\begin{array}{l}
4(-)^{n} e^{\imath s t / a} \operatorname{sh} s \operatorname{sh} t, \quad a=(n+1 / 2) \pi, \\
4 i(-)^{n+1} e^{\imath s t / a} \operatorname{ch} s \operatorname{ch} t, a=n \pi,
\end{array}\right. \\
& w_{1}(s)=\left\{\begin{array}{l}
1 / 4 \operatorname{sh}^{2} s, \\
1 / 4 \operatorname{ch}^{2} s,
\end{array} w_{2}(t)= \begin{cases}1 / 4 \operatorname{sh}^{2} t, & a=(n+1 / 2) \pi, \\
1 / 4 \operatorname{ch}^{2} t, & a=n \pi,\end{cases} \right. \\
& F(s, t)= \begin{cases}(-)^{n} e^{\imath s t / a} \operatorname{sign}(s) \operatorname{sign}(t), & a=(n+1 / 2) \pi, \\
(-)^{n+1} i e^{r s t / a}, & a=n \pi,\end{cases}
\end{aligned}
$$


yielding the same consequences as in the previous cases.

Requiring next (4.33), the formulas (4.34)-(4.36) apply unchanged to the present case. Thus the conclusion below (4.36) holds true for $H_{e}(1.15)$ as well.

For $N>1$ the reduction behavior is again the same as in the repulsive case, so it remains to consider $R_{2}$ for $a>\pi / 2 N$ with (3.5) in effect.

The relevant zeros of $c_{1}(-z)$ are given by (4.37), but now this leads to

$$
E\left(p_{k}^{(1)}, t\right)=(-)^{n_{k}} i^{k} \exp \left[\pi\left(n_{k}-1 / 2\right) t / a\right] B_{N-k}^{(N)}(i \operatorname{sh} t) .
$$

Therefore, instead of (4.39) we obtain, using (4.16),

$$
\begin{aligned}
R_{2} & =\frac{i}{a} \sum_{k=1}^{N} r_{k} \sum_{l=-n_{k}+1 / 2}^{n_{k}-1 / 2} f_{k,-l}^{(a)}(t) \otimes f_{k l}^{(a)}(t), \\
f_{k l}^{(a)}(t) & \equiv \exp (l \pi t / a) \psi_{N-k}^{(a)}(t) .
\end{aligned}
$$

Even so, it is obvious that (5.21) leads to the same conclusions as in the attractive case. In particular, (4.41) holds true.

\section{Appendix A. Proofs of Theorems 2.1-2.3}

This appendix contains the proofs of the three theorems in Section 2, and a few observations on how Theorems 2.2 and 2.3 can be extended to a quite general class of operators (containing the $A \Delta O$ s (1.13)-(1.15) in the pertinent special cases).

Proof of Theorem 2.1. We may write

$$
(\mathcal{F} \phi, \mathcal{F} \psi)_{1}=\frac{1}{2 \pi a} \lim _{R \rightarrow \infty} \int_{-\infty}^{\infty} d t \int_{-\infty}^{\infty} d t^{\prime} K\left(t, t^{\prime}\right) \int_{-R}^{R} d s f_{t . t^{\prime}}(s)
$$

where we have introduced

$$
\begin{aligned}
K\left(t, t^{\prime}\right) & \equiv \bar{\phi}(t) \psi\left(t^{\prime}\right) w_{2}(t)^{1 / 2} w_{2}\left(t^{\prime}\right)^{1 / 2}, \\
f_{t, t^{\prime}}(s) & \equiv w_{1}(s) E(s,-t) E\left(s, t^{\prime}\right) .
\end{aligned}
$$

(To verify this, one need only invoke (2.7) and Fubini's theorem.) Now we choose $R$ large enough so that the poles of $w_{1}(s)$ with $\operatorname{Im} s \in(0, \pi)$ are inside the rectangular contour $\Gamma$ connecting $-R, R, R+i \pi,-R+i \pi$ and $-R$. Then Cauchy's theorem yields

$$
\int_{\Gamma} d s f_{t, t^{\prime}}(s)=2 \pi i \mathcal{R}\left(t, t^{\prime}\right)
$$


where the residue sum is given by $(2.27)$.

Next, we observe that $f_{t, t^{\prime}}(s)$ (A.3) equals the product of an $i \pi$-periodic function and the plane wave $\exp i s\left(t^{\prime}-t\right) / a$, cf. (2.1) and (2.2). Thus we can rewrite (A.4) as

$$
\int_{-R}^{R} d s f_{t, t^{\prime}}(s)=\left[1-e^{\pi\left(t-t^{\prime}\right) / a}\right]^{-1}\left[2 \pi i \mathcal{R}\left(t, t^{\prime}\right)-\mathcal{B}_{R}\left(t, t^{\prime}\right)\right]
$$

where

$$
\mathcal{B}_{R}\left(t, t^{\prime}\right) \equiv\left(\int_{R}^{R+\imath \pi}+\int_{-R+i \pi}^{-R}\right) f_{t, t^{\prime}}(s) d s .
$$

We now take $s \rightarrow s+i \pi / 2$ in the integrals on the rhs of (A.6), and then $s \rightarrow-s$ in the second one. Since $w_{1}(s)$ is $i \pi$-periodic and even, this yields

$$
\begin{aligned}
\mathcal{B}_{R}\left(t, t^{\prime}\right)= & \int_{R-i \pi / 2}^{R+i \pi / 2} d s w_{1}\left(s+\frac{i \pi}{2}\right)\left[E\left(s+\frac{i \pi}{2},-t\right) E\left(s+\frac{i \pi}{2}, t^{\prime}\right)\right. \\
& \left.-E\left(-s+\frac{i \pi}{2},-t\right) E\left(-s+\frac{i \pi}{2}, t^{\prime}\right)\right] .
\end{aligned}
$$

We proceed by introducing the auxiliary function

$$
A\left(s, t, t^{\prime}\right) \equiv P\left(i e^{s}, e^{-t}\right) P\left(i e^{s}, e^{t^{\prime}}\right) .
$$

Using (2.6) and $i \pi$-periodicity of $A$ in $s$, we may rewrite $\mathcal{B}_{R}$ as

$$
\begin{aligned}
\mathcal{B}_{R}\left(t, t^{\prime}\right)= & \int_{R-i \pi / 2}^{R+\imath \pi / 2} d s w_{1}(s+i \pi / 2) e^{\pi\left(t-t^{\prime}\right) / 2 a} \\
& {\left[e^{\imath s\left(t^{\prime}-t\right) / a} A\left(s, t, t^{\prime}\right)-e^{\imath s\left(t-t^{\prime}\right) / a} A\left(s,-t,-t^{\prime}\right)\right] . }
\end{aligned}
$$

Now from (2.16) we have

$$
\begin{gathered}
w_{1}(s+i \pi / 2)=e^{-2 M_{1} s}+r(s), \\
r(s)=O\left(\exp \left(-\left(2 M_{1}+2\right) \operatorname{Re} s\right)\right), \quad \operatorname{Re} s \rightarrow \infty,
\end{gathered}
$$

where the bound is uniform for $\operatorname{Im} s \in \mathbb{R}$. Also, from (2.13) we readily obtain

$$
\begin{aligned}
A\left(s, t, t^{\prime}\right) & =\exp \left(2 M_{1} s\right) c_{2}(-t) c_{2}\left(t^{\prime}\right)+\rho\left(s, t, t^{\prime}\right), \\
\rho\left(s, t, t^{\prime}\right) & =O\left(\exp \left(\left(2 M_{1}-2\right) \operatorname{Re} s\right)\right), \quad \operatorname{Re} s \rightarrow \infty,
\end{aligned}
$$

where the bound is uniform for $\operatorname{Im} s, t$ and $t^{\prime}$ varying over compact subsets of $\mathbb{R}$. 
To exploit the above asymptotics, we expand (A.9) as follows:

$$
\begin{aligned}
\left(\text { A.14) } \mathcal{B}_{R}\left(t, t^{\prime}\right)=\right. & e^{\pi\left(t-t^{\prime}\right) / 2 a} \int_{R-\imath \pi / 2}^{R+i \pi / 2} d s \sum_{j=1}^{4} b_{\jmath}\left(s, t, t^{\prime}\right), \\
(\text { A.15 }) & b_{1} \equiv-i \sin \left(s\left(t-t^{\prime}\right) / a\right)\left[c_{2}(-t) c_{2}\left(t^{\prime}\right)+c_{2}(t) c_{2}\left(-t^{\prime}\right)\right], \\
(\text { A.16) } & b_{2} \equiv-i \sin \left(s\left(t-t^{\prime}\right) / a\right) \rho_{+}\left(s, t, t^{\prime}\right), \\
(\text { A.17) } & b_{3} \equiv \cos \left(s\left(t-t^{\prime}\right) / a\right)\left[c_{2}(-t) c_{2}\left(t^{\prime}\right)-c_{2}(t) c_{2}\left(-t^{\prime}\right)\right], \\
(\text { A.18) } & b_{4} \equiv \cos \left(s\left(t-t^{\prime}\right) / a\right) \rho_{-}\left(s, t, t^{\prime}\right), \\
\left(\text { A.19) } \rho_{\delta}\left(s, t, t^{\prime}\right) \equiv\right. & {\left[\exp \left(-2 M_{1} s\right)+r(s)\right]\left[\rho\left(s, t, t^{\prime}\right)+\delta \rho\left(s,-t,-t^{\prime}\right)\right] } \\
& +r(s) \exp \left(2 M_{1} s\right)\left[c_{2}(-t) c_{2}\left(t^{\prime}\right)+\delta c_{2}(t) c_{2}\left(-t^{\prime}\right)\right], \quad \delta=+,-.
\end{aligned}
$$

Each of the terms in the sum on the rhs of (A.14) is a $C^{\infty}$-function of $t$ and $t^{\prime}$ that vanishes for $t=t^{\prime}$. Thus the integrals

$$
\begin{aligned}
I_{\jmath}(R) \equiv & -\frac{1}{2 \pi a} \int_{-\infty}^{\infty} d t \int_{-\infty}^{\infty} d t^{\prime} K\left(t, t^{\prime}\right) e^{\pi\left(t-t^{\prime}\right) / 2 a} \\
& \cdot\left[1-e^{\pi\left(t-t^{\prime}\right) / a}\right]^{-1} \int_{R-\imath \pi / 2}^{R+\imath \pi / 2} d s b_{\jmath}\left(s, t, t^{\prime}\right), \quad j=1, \ldots, 4,
\end{aligned}
$$

are well defined, and in view of (A.1) and (A.5) it suffices to substantiate the limits

$$
\begin{aligned}
& \lim _{R \rightarrow \infty} I_{1}(R)=(\phi, \psi)_{1}, \\
& \lim _{R \rightarrow \infty} I_{\jmath}(R)=0, \quad j=2,3,4 .
\end{aligned}
$$

In order to prove (A.21), we use (A.20) and (A.15) to calculate

$$
I_{1}(R)=\frac{1}{2 \pi} \int_{-\infty}^{\infty} d t \int_{-\infty}^{\infty} d t^{\prime} K\left(t, t^{\prime}\right) \frac{\sin R\left(t-t^{\prime}\right) / a}{t-t^{\prime}}\left[c_{2}(-t) c_{2}\left(t^{\prime}\right)+c_{2}(t) c_{2}\left(-t^{\prime}\right)\right] .
$$

Recalling the well-known tempered distribution limit

$$
\lim _{c \rightarrow \infty} \frac{\sin c x}{x}=\pi \delta(x)
$$

and using also (2.14), we now deduce (A.21). (Observe that $K\left(t, t^{\prime}\right)(\mathrm{A} .2)$ is a $C_{0}^{\infty}$-function.)

We continue by studying $I_{2}(R)$. The integral is proportional to

$$
\int_{-\infty}^{\infty} d t \int_{-\infty}^{\infty} d t^{\prime} K\left(t, t^{\prime}\right) \frac{t-t^{\prime}}{\operatorname{sh}\left(\pi\left(t-t^{\prime}\right) / 2 a\right)} \int_{R-\imath \pi / 2}^{R+\imath \pi / 2} d s \frac{\sin \left(s\left(t-t^{\prime}\right) / a\right)}{t-t^{\prime}} \rho_{+}\left(s, t, t^{\prime}\right)
$$


The integrand of the $s$-integral can be estimated by using

$$
\begin{aligned}
\left|\frac{\sin s\left(t-t^{\prime}\right) / a}{\left(t-t^{\prime}\right) / a}\right| & =\frac{1}{2}\left|\int_{-s}^{s} d x e^{i x\left(t-t^{\prime}\right) / a}\right| \leq|s| e^{\left|t-t^{\prime}\right||\operatorname{Im} s| / a}, \\
\rho_{+}\left(s, t, t^{\prime}\right) & =O(\exp (-2 \operatorname{Re} s)), \quad \operatorname{Re} s \rightarrow \infty
\end{aligned}
$$

where the latter bound is uniform for $\operatorname{Im} s, t$ and $t^{\prime}$ in compacts, cf. (A.19), (A.11) and (A.13). Hence we easily deduce (A.22) for $j=2$.

Consider next $I_{3}(R)$. This integral is proportional to

$$
\int_{-\infty}^{\infty} d t \int_{-\infty}^{\infty} d t^{\prime} K\left(t, t^{\prime}\right) \frac{\cos R\left(t-t^{\prime}\right) / a}{t-t^{\prime}}\left[c_{2}(-t) c_{2}\left(t^{\prime}\right)-c_{2}(t) c_{2}\left(-t^{\prime}\right)\right]
$$

Thus its integrand equals $\cos \left(R\left(t-t^{\prime}\right) / a\right)$ times a function in $C_{0}^{\infty}\left(\mathbb{R}^{2}\right)$. Its $R \rightarrow \infty$ limit then vanishes by virtue of the Riemann-Lebesgue lemma.

Finally, we take $j=4$ in (A.20), obtaining an integral proportional to

$$
\int_{-\infty}^{\infty} d t \int_{-\infty}^{\infty} d t^{\prime} K\left(t, t^{\prime}\right) \frac{t-t^{\prime}}{\operatorname{sh}\left(\pi\left(t-t^{\prime}\right) / 2 a\right)} \int_{R-\imath \pi / 2}^{R+\imath \pi / 2} d s \cos \left(s\left(t-t^{\prime}\right) / a\right) \frac{\rho_{-}\left(s, t, t^{\prime}\right)}{t-t^{\prime}}
$$

Now $\rho_{-}$vanishes for $t=t^{\prime}$, so we have

$$
\begin{aligned}
\left|\frac{\rho_{-}\left(s, t, t^{\prime}\right)}{t-t^{\prime}}\right| & =\left|\frac{1}{t-t^{\prime}} \int_{t}^{t^{\prime}} d x \partial_{3} \rho_{-}(s, t, x)\right| \\
& \leq \max _{\left(t, t^{\prime}, \theta\right) \in \operatorname{supp} K \times[0,1]}\left|\partial_{3} \rho_{-}\left(s, t, t+\theta\left(t^{\prime}-t\right)\right)\right| .
\end{aligned}
$$

Recalling the definitions (A.12) and (A.8), one readily checks that the bound (A.13) also holds for the $t^{\prime}$-partial of $\rho\left(s, t, t^{\prime}\right)$, uniformly for $\operatorname{Im} s, t, t^{\prime}$ in compacts. Thus the rhs of (A.30) is $O(\exp (-2 \operatorname{Re} s))$ for $\operatorname{Re} s \rightarrow \infty$, uniformly for Im $s$ in compacts. Clearly, this entails that (A.29) has limit 0 for $R \rightarrow \infty$, completing the proof of Theorem 2.1.

Proof of Theorem 2.2. Letting $\phi, \psi \in \mathcal{C}_{2}$, we obtain from (2.42) and (2.32) the equalities

$$
\begin{aligned}
& \left(A_{1} \mathcal{F} \phi, \mathcal{F} \psi\right)_{1}=\left(\mathcal{F} \mathcal{M}_{2} \phi, \mathcal{F} \psi\right)_{1}=\left(\mathcal{M}_{2} \phi, \psi\right)_{2}+\left(\mathcal{M}_{2} \phi, R_{2} \psi\right)_{2} \\
& \left(\mathcal{F} \phi, A_{1} \mathcal{F} \psi\right)_{1}=\left(\mathcal{F} \phi, \mathcal{F} \mathcal{M}_{2} \psi\right)_{1}=\left(\phi, \mathcal{M}_{2} \psi\right)_{2}+\left(\phi, R_{2} \mathcal{M}_{2} \psi\right)_{2}
\end{aligned}
$$

Now assume first $R_{2}=0$. Then we read off from (A.31) and (A.32) that $A_{1}$ is symmetric on $\mathcal{P}_{1}$. Since $\mathcal{C}_{2}$ is a core for the self-adjoint multiplication operator 
$\mathcal{M}_{2}$ (by virtue of Nelson's analytic vector theorem, cf. [49]), and since $\mathcal{F}$ is an isometry when $R_{2}=0$, we deduce that $A_{1}$ is e.s.a. on $\mathcal{P}_{1}$.

It remains to show that $A_{1}$ is not symmetric when $R_{2} \neq 0$. In view of (A.31) and (A.32), this will follow once we prove

$$
\left(\mathcal{M}_{2} \phi, \mathcal{R}_{2} \psi\right)_{2} \neq\left(\phi, R_{2} \mathcal{M}_{2} \psi\right)_{2}
$$

for suitable vectors $\phi, \psi \in \mathcal{C}_{2}$. To demonstrate the existence of such pairs, we assume that the two inner products are equal for all $\phi, \psi \in \mathcal{C}_{2}$ and derive a contradiction.

Indeed, we have $\mathcal{C}_{2}=\left(\mathcal{M}_{2} \pm i\right)\left(\mathcal{C}_{2}\right)$, so the assumption just made entails

$$
\left(\chi, R_{2}\left(\mathcal{M}_{2}-i\right)^{-1} \eta\right)_{2}=\left(\left(\mathcal{M}_{2}+i\right)^{-1} \chi, R_{2} \eta\right)_{2}
$$

for all $\chi, \eta \in \mathcal{C}_{2}$. Therefore, the bounded normal operator $\left(\mathcal{M}_{2}-i\right)^{-1}$ and the bounded self-adjoint operator $R_{2}$ commute. Hence $\left(\mathcal{M}_{2}-i\right)^{-1}$ leaves the $L$-dimensional range $\mathcal{R}_{2}$ invariant. As a consequence, $\left(\mathcal{M}_{2}-i\right)^{-1}$ has $L$ eigenvectors. Since the normal operator $\left(\mathcal{M}_{2}-i\right)^{-1}$ has solely continuous spectrum, we deduce $L=0$, contradicting $R_{2} \neq 0$.

With an eye on the concrete special cases studied in Sections 3-5 we add a comment on Theorem 2.2 and the proof just given. Let us reconsider the intertwining relation (2.42), which we used to define a Hilbert space operator $A_{1}$ by starting from a specific self-adjoint multiplication operator $\mathcal{M}_{2}$. When we now reinterpret $\mathcal{M}_{2}$ as multiplication by an arbitrary real-valued smooth function $m(t)$, then we can still use (2.42) to define an operator $A_{1}$ on $\mathcal{P}_{1}$, provided the kernel assumption in Theorem 2.2 is met when $R_{2} \neq 0$. (Of course, the action of this pull-back operator will not generally be equal to that of an $\mathrm{A} \Delta \mathrm{O}$.) The point we wish to make here is that Theorem 2.2 applies unchanged to this more general operator $A_{1}$, provided $\mathcal{M}_{2}$ has purely continuous spectrum. (For instance, it suffices that $m^{\prime}(t)$ vanishes only on a discrete set.) Indeed, the above proof holds true verbatim.

Proof of Theorem 2.3. It suffices to prove

$$
\lim _{T \rightarrow \pm \infty} F_{ \pm}(T)=0, \quad F_{ \pm}(T) \equiv\left\|\left(e^{i T A_{1}} J e^{-\imath T M_{2}}-W_{ \pm}\right) \phi\right\|_{1},
$$

where the operators $W_{ \pm}$are defined by (2.53), and where $\phi$ belongs to the dense subspace $\mathcal{C}_{2}$ defined below (2.20). (Indeed, the operators involved are isometric.) To this end we begin by noting that we have

$$
F_{ \pm}(T)^{2}=\left\|\left(J-W_{ \pm}\right) e^{-i T M_{2}} \phi\right\|_{1}^{2},
$$


by virtue of (2.42). From the above definitions we then obtain

$$
F_{ \pm}(T)^{2}=\frac{1}{2 \pi a} \int_{-\infty}^{\infty} d s\left|\int_{-\infty}^{\infty} d t \phi(t) \exp (i[s t / a-2 T \operatorname{ch}(\pi t / a)]) D_{ \pm}(s, t)\right|^{2}
$$

with difference functions given by

$D_{ \pm}(s, t) \equiv 1-w_{1}(s)^{1 / 2} P\left(e^{s}, e^{t}\right) w_{2}(t)^{1 / 2} \sigma_{ \pm}(t)\left[c_{2}(|t|) / c_{2}(-|t|)\right]^{\mp 1 / 2} \operatorname{sign}\left(c_{2}(0)\right)$.

Using the Riemann-Lebesgue lemma, one readily checks that for $s$ fixed the $t$-integral converges to 0 for $T \rightarrow \pm \infty$. To exploit this, we split up the $s$-integration region in three subsets, viz., $s \in[-1,1], \pm s \in(1, \infty)$. A routine application of the dominated convergence theorem then shows that the $s$-integral over $[-1,1]$ converges to 0 for $T \rightarrow \pm \infty$.

Consider now the $s$-integral over $(1, \infty)$. We have

$$
w_{1}(s)^{1 / 2} P\left(e^{s}, e^{t}\right)=c_{2}(t)+R^{(+)}(s, t)
$$

where the remainder satisfies

$$
R^{(+)}(s, t)=O\left(e^{-2 s}\right), \quad s \rightarrow \infty,
$$

uniformly for $t$ in compacts. (Recall (2.1), (2.13) and (2.16) to see this.) Substituting (A.39) in (A.38), we split up the $t$-integrand by writing $D_{ \pm}(s, t)$ as the sum of the leading $s \rightarrow \infty$ asymptotics

$$
L_{ \pm}^{(+)}(t) \equiv 1-\frac{c_{2}(t) \sigma_{ \pm}(t)}{\left[c_{2}(t) c_{2}(-t)\right]^{1 / 2}}\left(\frac{c_{2}(|t|)}{c_{2}(-|t|)}\right)^{\mp 1 / 2} \operatorname{sign}\left(c_{2}(0)\right),
$$

and a remainder involving $R^{(+)}(s, t)$. The uniform bound (A.40) can then be used to dominate the pointwise convergence to 0 of the $s$-integrand for the remainder function.

We are therefore left with estimating

$$
\int_{1}^{\infty} d s\left|\int_{-\infty}^{\infty} d t \phi(t) L_{\delta}^{(+)}(t) \Phi(s, t)\right|^{2}, \quad \delta=+,-,
$$

where we have introduced

$$
\Phi(s, t) \equiv[s-2 \pi T \operatorname{sh}(\pi t / a)]^{-1} \partial_{t} \exp (i(s t / a-2 T \operatorname{ch}(\pi t / a))) .
$$


The crux is now that the above phase conventions and the definition (2.50) of $\sigma_{ \pm}(t)$ ensure that $L_{+}^{(+)}(t)$ vanishes for $t>0$ and $L_{-}^{(+)}(t)$ for $t<0$. On the remaining $t$-interval the function in square brackets in (A.43) (with $s>1$ ) does not vanish for $T>0$ and $T<0$, resp. Hence we can integrate by parts and estimate in the obvious way to deduce that (A.42) converges to 0 for $T \rightarrow \delta \infty, \delta=+,-$.

Finally, we can handle the $s$-integral over $(-\infty,-1)$ in substantially the same way by using

$$
\begin{aligned}
w_{1}(s)^{1 / 2} P\left(e^{s}, e^{t}\right) & =\sigma c_{2}(-t)+R^{(-)}(s, t), \\
R^{(-)}(s, t) & =O\left(e^{2 s}\right), \quad s \rightarrow-\infty
\end{aligned}
$$

Indeed, setting

$$
L_{ \pm}^{(-)}(t) \equiv 1-\frac{\sigma c_{2}(-t) \sigma_{ \pm}(t)}{\left[c_{2}(t) c_{2}(-t)\right]^{1 / 2}}\left(\frac{c_{2}(|t|)}{c_{2}(-|t|)}\right)^{\mp 1 / 2} \operatorname{sign}\left(c_{2}(0)\right)
$$

the function $L_{+}^{(-)}(t)$ vanishes for $t<0$ and $L_{-}^{(-)}(t)$ for $t>0$. Therefore the proof of (A.35) is complete.

We conclude this appendix with some remarks concerning generalizations of Theorem 2.3, cf. also the comment made after the proof of Theorem 2.2. First, when we generalize the operator $\mathcal{M}_{2}$ to the operator $\mathcal{M}_{2}(\lambda)$ of multiplication by $2 \operatorname{ch}(\lambda t), \lambda>0$, then Theorem 2.3 and its proof can be adapted in an obvious way to the pull-back operator $A_{1}(\lambda)$ defined on $\mathcal{P}_{1}$ by

$$
A_{1}(\lambda) \mathcal{F}=\mathcal{F} \mathcal{M}_{2}(\lambda)
$$

(As is detailed in Sections 3 and 4, for the repulsive and attractive transforms the action of $A_{1}(1)$ equals that of $H_{r}$ and $H_{a}$, resp.) More generally, whenever the multiplier function $m(t)$ is real-valued, smooth, and satisfies

$$
m^{\prime}(t)>0, t>0, \quad m^{\prime}(t)<0, t<0
$$

the above proof applies with obvious changes. Therefore, all of these dynamics lead to the same wave maps $W_{ \pm}(2.53)$ and $S$-operator $S_{2}(2.54)$.

Consider now the operators $\tilde{\mathcal{M}}_{2}(\lambda)$ of multiplication by $2 \operatorname{sh}(\lambda t), \lambda>0$, and their pull-backs $\tilde{A}_{1}(\lambda)$ under $\mathcal{F}$. (The Hamiltonian $H_{e}$ corresponds to $\tilde{A}_{1}(1)$, cf. Section 5.) Inspecting the above proof, one sees that it breaks down. Indeed, the function $\Phi(s, t)$ (A.43) is replaced by

$$
\tilde{\Phi}(s, t) \equiv[s-2 a \lambda T \operatorname{ch}(\lambda t)]^{-1} \partial_{t} \exp (i(s t / a-2 T \operatorname{sh}(\lambda t))) .
$$


Hence the square-bracket function has different vanishing characteristics for $t<0$.

In point of fact, it is not hard to see that the wave maps are different for the latter class of dynamics, so that the above proof must break down. Specifically, they are now given by

$$
\begin{aligned}
& \tilde{W}_{+} \equiv \mathcal{F}\left[c_{2}(t) / c_{2}(-t)\right]^{-1 / 2} \operatorname{sign}\left(c_{2}(0)\right), \\
& \tilde{W}_{-} \equiv \mathcal{F} \sigma\left[c_{2}(t) / c_{2}(-t)\right]^{1 / 2} \operatorname{sign}\left(c_{2}(0)\right),
\end{aligned}
$$

yielding an $S$-operator

$$
\tilde{S}_{2} \equiv \tilde{W}_{+}^{*} \tilde{W}_{-}=\sigma \frac{c_{2}(t)}{c_{2}(-t)}
$$

To check this, one need only retrace the steps of the above proof. Then one concludes that the leading $s \rightarrow \infty$ asymptotics $\tilde{L}_{+}^{(+)}(t)$ vanishes both for $t>0$ and for $t<0$; similarly, $\tilde{L}_{-}^{(-)}(t)$ vanishes identically; since the square-bracket function in (A.49) neither vanishes for $s>1, T<0, t \in \mathbb{R}$ nor for $s<-1, T\rangle$ $0, t \in \mathbb{R}$, it supplies the necessary domination for the remaining cases.

Once more, the $S$-matrix $\tilde{S}_{2}$ (A.52) can be physically understood from the asymptotics (2.55) of the generalized eigenfunction $F(s, t)$ : A particle whose time evolution is governed by $\tilde{A}_{1}(\lambda)$ moves from left to right not only for $t>0$ (just as for $A_{1}(\lambda)$-evolution), but also for $t<0$ (in contrast to $A_{1}(\lambda)$-evolution.) Therefore, the phase change (A.52) is the same as the phase change (2.54) for $t>0$, whereas it is the opposite for $t<0$.

\section{References}

[1] Ruijsenaars, S. N. M. and Schneider, H.. A new class of integrable systems and its relation to solitons, Ann. Phys. (N.Y.), 170 (1986), 370-405.

[2] Ruijsenaars, S. N. M., Complete integrability of relativistic Calogero-Moser systems and elliptic function identities, Comm. Math. Phys., 110 (1987), 191-213.

[3] - Systems of Calogero-Moser type, in "Proceedings of the 1994 Banff summer school Partıcles and fields", CRM series in mathematical physics, G. Semenoff, L. Vinet, Eds., pp. 251-352, Springer, New York, 1999.

[4] Antonov, A., Hasegawa, K. and Zabrodin, A., On trigonometric intertwining vectors and non-dynamical $R$-matrix for the Ruijsenaars model, Nucl. Phys. B, 503 (1997), 747-770.

[5] Arutyunov, G. E., Frolov, S. A. and Medvedev, P. B., Elliptic Ruijsenaars-Schneider model via the Poisson reduction of the affine Heisenberg double, J. Phys. A, 30 (1997), 5051-5063.

[6] Arutyunov, G. E., Chekhov, L. O. and Medvedev, P. B., Elliptic Ruijsenaars-Schneider model from the cotangent bundle over the two-dimensional current group, J. Math. Phys., 38 (1997), 5682-5689. 
[7] Arutyunov, G. E., Chekhov, L. O. and Frolov, S. A., $R$-matrix quantization of the elliptic Ruijsenaars-Schneider model, Comm. Math. Phys., 192 (1998), 405-432.

[8] Avan, J. and Rollet, G., The classical $r$-matrix for the relativistic Ruijsenaars-Schneider system, Phys. Lett. A, 212 (1996), 50-54.

[9] Braden, H. W. and Hone, A. N. W., Affine Toda solitons and systems of Calogero-Moser type, Phys. Lett. B, 380 (1996), 296-302.

[10] Braden, H. W. and Sasaki, R., The Ruijsenaars-Schneider model, Prog. Theor. Phys., 97 (1997), 1003-1017.

[11] Braden, H. W., Marshakov, A., Mironov, A. and Morozov, A., The RuijsenaarsSchneider model in the context of Seiberg-Witten theory, Nucl. Phys. B, 558 (1999), 371-390.

[12] Chalykh, O. A., The duality of the generalized Calogero and Ruijsenaars problems, Russ. Math. Surveys, 52 (1997), 1289-1291.

[13] Chen, K., Fan, H., Hou, B., Shi, K., Yang, W. and Yue, R., Elliptic RuijsenaarsSchneider and Calogero-Moser models represented by Sklyanin algebra and $s l(n)$ Gaudin algebra, Prog. Theor. Phys. Suppl., 135 (1999), 149-165.

[14] Chen, K., Hou, B., Yang, W. and Zhen, Y., Nondynamical $r$-matrix structure of the sl(2) trigonometric Ruijsenaars-Schneider model, Chinese Phys. Lett., 16 (1999), 1-3.

[15] Cherednik, I., Elliptic quantum many-body problem and double affine KniznikZamolodchikov equation, Comm. Math. Phys., 169 (1995), 441-461.

[16] _ Inverse Harish-Chandra transform and difference operators, Int. Math. Res. Notices, no. 15 (1997), 733-750.

[17] van Diejen, J. F. and Vinet, L., The quantum dynamics of the compactified trigonometric Ruijsenaars-Schneider model, Comm. Math. Phys., 197 (1998), 33-74.

[18] Felder, G. and Varchenko, A., Elliptic quantum groups and Ruijsenaars models, J. Stat. Phys., 89 (1997), 963-980.

[19] - Algebraic integrability of the two-body Ruijsenaars operator, Funct. Anal. Appl., 32 (1998), 81-92.

[20] Gorsky, A., Integrable many-body systems in the field theories, Theor. Math. Phys., 103 (1995), 681-700.

[21] Gorsky, A. and Nekrasov, N., Relativistic Calogero-Moser model as gauged WZW theory, Nucl. Phys. B, 436 (1995), 582-608.

[22] Hasegawa, K., Ruijsenaars' commuting difference operators as commuting transfer matrices, Comm. Math. Phys., 187 (1997), 289-325.

[23] Hikami, K., Affine Hecke algebra, Macdonald polynomials, and quantum many-body systems, J. Phys. A, 29 (1996), L281-L287.

[24] Hikami, K. and Komori, Y., Integrability, fusion, and duality in the elliptic Ruijsenaars model, Mod. Phys. Lett. A, 12 (1997), 751-761.

[25] - Diagonalization of the elliptic Ruijsenaars model. Correspondence with the Belavin model, Europ. Phys. J. B, 5 (1998), 583-588.

[26] Hou, B., Shi, K., Wang, Y. and Zhao, L., Ruijsenaars-Macdonald-type difference operators from $Z_{n}$ Belavin model with open boundary conditions, J. Phys. A, 31 (1998), 5911-5923.

[27] Itoyama, H. and Morozov, A., Prepotential and the Seiberg-Witten theory, Nucl. Phys. $B, 491$ (1997), 529-573.

[28] Jurčo, B. and Schupp, P., Adler-Kostant-Symes scheme for face and Calogero-MoserSutherland-type models, J. Math. Phys., 39 (1998), 3577-3588.

[29] Komori, Y., Notes on the elliptic Ruijsenaars operators, Lett. Math. Phys., 46 (1998), $147-155$.

[30] Komori, Y. and Hikami, K., Quantum integrability of the generalized elliptic Ruijsenaars models, J. Phys. A, 30 (1997), 4341-4364.

[31] Konno, H., Relativistic Calogero-Sutherland model: Spin generalization, quantum affine symmetry and dynamical correlation functions, J. Phys. A, 29 (1996), L191-L198. 
[32] Konno, H., Dynamical correlation functions and finite-size scaling in the RuijsenaarsSchneider model, Nucl. Phys. B, 473 (1996), 579-600.

[33] Krichever, I. and Zabrodin, A., Spin generalization of the Ruijsenaars-Schneider model, non-abelian 2D Toda chain and representations of Sklyanin algebra, Russ. Math. Surveys, 50 (1995), 1101-1150.

[34] Kuznetsov, V. B. and Sklyanin, E. K., Separation of variables for the $A_{2}$ Ruijsenaars model and a new integral representation for the $A_{2}$ Macdonald polynomials, J. Phys. A, 29 (1998), 2779-2804.

[35] Kuznetsov, V. B., Nijhoff, F. W. and Sklyanin, E. K., Separation of variables for the Ruijsenaars system, Comm. Math. Phys., 189 (1997), 855-877.

[36] Kuznetsov, V. B. and Sklyanin, E. K., On Bäcklund transformations for many-body systems, J. Phys. A, 31 (1998), 2241-2251.

[37] Nekrasov, N., Holomorphic bundles and many-body systems, Comm. Math. Phys., 180 (1996), 587-603.

[38] - Five-dimensional gauge theories and relativistic integrable systems, Nucl. Phys. $B, 531$ (1998), 323-344.

[39] Nijhoff, F. W., Ragnisco, O. and Kuznetsov, V. B., Integrable time-discretisation of the Ruijsenaars-Schneider model, Comm. Math. Phys., 176 (1996), 681-700.

[40] Nijhoff, F. W., Kuznetsov, V. B. and Sklyanin, E. K., Dynamical $r$-matrix for the elliptic Ruijsenaars-Schneider system, J. Phys. A, 29 (1996), L333-L340.

[41] Ragnisco, O. and Suris, Y. B., Integrable discretizations of the spin RuijsenaarsSchneider models, J. Math. Phys., 38 (1997), 4680-4691.

[42] Ruijsenaars, S. N. M., Integrable particle systems vs solutions to the KP and 2D Toda equations, Ann Phys. (NY), 256 (1997), 226-301.

[43] Suris, Y. B., Why is the Ruijsenaars-Schneider hierarchy governed by the same $R$ operator as the Calogero-Moser one?, Phys. Lett. A, 225 (1997), 253-262.

[44] Zabrodin, A. V., Hirota equation and Bethe Ansatz, Theor. Math. Phys., 116 (1998), $782-820$.

[45] Ruijsenaars, S. N. M., Generalized Lamé functions. II. Hyperbolic and trigonometric specializations, J. Math. Phys., 40 (1999), 1595-1626.

i46] Titchmarsh, E. C., Eigenfunction expansions associated with second-order differential equations. Part I, Oxford University Press, Oxford, 1962.

[47] Dunford, N. and Schwartz, J. T., Lınear operators. Part II: Spectral theory, Interscience Publ., New York, 1963.

[48] Berezanskii, Ju. M., Expansıons in ergenfunctıons of self-adjoint operators, Transl. Math. Monogr., 17 (1968).

[49] Reed, M. and Simon, B., Methods of modern mathematıcal physıcs. II. Fourier analysıs, self-adjorntness, Academic Press, New York, 1975.

[50] Fulling, S. A., Aspects of quantum field theory in curved space-time, London Math. Soc. Stud. Texts, 17, Cambridge University Press, Cambridge, 1989.

[51] Jirari, A., Second-order Sturm-Liouville difference equations and orthogonal polynomials, Mem. AMS, 113, no. 542, (1995).

[52] Ruijsenaars, S. N. M., Generalized Lamé functions. I. The elliptic case, J. Math. Phys., 40 (1999), 1627-1663.

[53] - Relativistic Lamé functions: The special case $g=2$, J. Phys. A, 32 (1999), 1737-1772.

[54] Gasper, G. and Rahman, M., Basıc hypergeometrıc series, Encyclopedia Math. Appl., 35 (1990).

[55] Ruijsenaars, S. N. M., Finite-dimensional soliton systems, in "Integrable and superıntegrable systems" (B. Kupershmidt, Ed.), pp. 165-206, World Scientific, Singapore, 1990.

[56] - A generalized hypergeometric function satisfying four analytic difference equations of Askey-Wilson type, Comm. Math. Phys., 206 (1999), 639-690.

[57] Reed, M. and Simon, B., Methods of modern mathematıcal physıcs. III. Scattering theory, Academic Press, New York, 1979. 
[58] Reed, M. and Simon, B., Methods of modern mathematıcal physıcs. I. Functıonal analysis, Academic Press, New York, 1972.

[59] Ruijsenaars, S. N. M., First order analytic difference equations and integrable quantum systems, J. Math. Phys., 38 (1997), 1069-1146. 
